MATHEMATICS OF COMPUTATION

Volume 74, Number 249, Pages 123-151

S $0025-5718(04) 01680-1$

Article electronically published on July 20, 2004

\title{
NUMERICAL TREATMENT OF REALISTIC BOUNDARY CONDITIONS FOR THE EDDY CURRENT PROBLEM IN AN ELECTRODE VIA LAGRANGE MULTIPLIERS
}

\author{
ALFREDO BERMÚDEZ, RODOLFO RODRÍGUEZ, AND PILAR SALGADO
}

\begin{abstract}
This paper deals with the finite element solution of the eddy current problem in a bounded conducting domain, crossed by an electric current and subject to boundary conditions appropriate from a physical point of view. Two different cases are considered depending on the boundary data: input current density flux or input current intensities. The analysis of the former is an intermediate step for the latter, which is more realistic in actual applications. Weak formulations in terms of the magnetic field are studied, the boundary conditions being imposed by means of appropriate Lagrange multipliers. The resulting mixed formulations are analyzed depending on the regularity of the boundary data. Finite element methods are introduced in each case and error estimates are proved. Finally, some numerical results to assess the effectiveness of the methods are reported.
\end{abstract}

\section{INTRODUCTION}

The aim of this paper is to analyze finite element methods to solve the eddy current problem in a conducting bounded domain. In particular, we consider the case of an electrode crossed by an alternating current and subject to boundary conditions which are non-standard, but realistic from the point of view of applications.

Numerical solution of the eddy current model became an important research area in recent years because of many applications in electrical engineering (see for instance [10] and references therein). In particular, the present work is motivated by the need of a three-dimensional model to study the behavior of the electrodes in a metallurgical electric furnace. A considerable number of mathematical models and computer codes have been designed for this purpose, but in most cases they are based on cylindrical symmetry (see for instance [5, 6, 16]). All of these models give

Received by the editor October 15, 2002 and, in revised form, July 16, 2003.

2000 Mathematics Subject Classification. Primary 78M10, 65N30.

Key words and phrases. Low-frequency harmonic Maxwell equations, eddy current problems, finite element computational electromagnetism, Lagrange multipliers.

This work was partially supported by Programa de Cooperación Científica con Iberoamérica, Ministerio de Educación y Ciencia, Spain.

The first author was partially supported by Xunta de Galicia grant PGIDT00PXI20701PR (Spain).

The second author was partially supported by FONDAP in Applied Mathematics (Chile).

The third author was partially supported by FEDER-CICYT grant 1FD97.0280 (Spain).

(C)2004 American Mathematical Society 
valuable information on important electrode parameters; however, the axisymmetric assumption makes it necessary to neglect aspects such as the electromagnetic effect caused on one electrode by the others (the so-called "proximity effect") or the phase-difference of the input electric current in the electrode.

A finite element method to solve the eddy current problem in the whole furnace is studied in 7 . In this case, the computational bounded three-dimensional domain includes not only conductors (the electrodes) but dielectrics as well (the air). This leads to the use of a scalar magnetic potential and Lagrange multipliers. However, the model presented in that paper is highly complex and its numerical solution takes a lot of computer time. This is why it is useful to have simpler models to describe separate components of the whole system. In the present work, we solve the eddy current model in a domain which includes only one electrode (see Figure 1 in the next section). Thus, we get an important saving in computational effort while still being able to take into account important three-dimensional aspects, although not the presence of the two other electrodes.

While several papers deal with the eddy current problem in the whole space (see for instance [9, 12, 15, 17, 18]), the number of papers concerning analysis in a bounded domain is much smaller, due in part to the difficulty of handling realistic boundary conditions. Essential and natural boundary conditions related to the tangential component of the electric and magnetic fields are considered in [2, [7, and [1. However, these conditions are not directly related to the physical data which, in the case of an electrode, usually reduces to the input current intensity on the boundary of the conducting domain.

To our knowledge, a description of more general boundary conditions can be found only in [11. However, the main goal of that paper is concerned with topological aspects of the domain and with the introduction of elements of homology to correctly define these boundary conditions, but not with the mathematical study of corresponding variational formulations and their discretization.

In the present paper, we propose and analyze finite element methods to solve the eddy current model in a conducting bounded domain, including boundary conditions appropriate from a physical point of view. In particular, we consider a formulation in terms of the magnetic field and impose boundary conditions related either to the current density flux or to the current intensities entering the conducting domain.

Our main goal is to analyze and solve the last case, because current intensities are usually the only known data in real problems. However, we consider first the eddy current problem with current density flux as boundary data, since this allows us to present the results in a simpler form. Nevertheless, the analysis of this case can be of interest on its own, for instance in coupled problems where the current density flux is the output of other computations.

To impose the different boundary conditions, we introduce Lagrange multipliers and study the resulting mixed formulations. Concerning the discretizations of the problems, the magnetic field is approximated by Nédélec edge finite elements, while the Lagrange multipliers are in principle discretized by continuous piecewise linear functions.

We also prove that the Lagrange multiplier is a physically relevant field: an electric surface potential defined on the boundary of the conducting domain. This is why it turns out to be interesting to obtain error estimates for this magnitude, 
too. To this aim, we consider an alternative discretization of the Lagrange multiplier by piecewise constant functions, valid under a further mild regularity assumption on the current density flux.

The outline of the paper is as follows. In Section 2, we recall the eddy current model and define two sets of boundary conditions under which the electromagnetic problem is well posed. Section 3 is devoted to obtaining weak formulations by using the input current density flux as boundary data and to proving the existence and uniqueness of the solution. Then, in Section 4, we analyze the problem when the boundary data reduces to the input current intensity. Numerical discretizations of these problems are described in Section 5 where error estimates are obtained under mild regularity assumption on the solution. Finally, in Section 6 , we report numerical results for a test problem with known analytical solution which allow us to assess the performance of the methods.

Some of the results of this paper have been announced in reference [8].

\section{The EDDY CURRENT PROBLEM}

Eddy currents are usually modeled by the low-frequency harmonic Maxwell equations. We are interested in solving the problem in a bounded conducting domain $\Omega$ (the electrode) crossed by an alternating electric current of angular frequency $\omega$. In this case the model reduces to

$$
\begin{aligned}
\operatorname{curl} \mathbf{H}=\mathbf{J} & \text { in } \Omega, \\
\mathrm{i} \omega \mu \mathbf{H}+\operatorname{curl} \mathbf{E}=\mathbf{0} & \text { in } \Omega, \\
\mathbf{J}=\sigma \mathbf{E} & \text { in } \Omega,
\end{aligned}
$$

where $\mathbf{H}, \mathbf{E}$ and $\mathbf{J}$ are the complex amplitudes of the magnetic field, electric field and current density, respectively (see for instance [10]). Throughout the paper, we use boldface letters to denote vector fields and variables, as well as vector-valued operators.

The coefficients $\mu$ and $\sigma$ are the magnetic permeability and the electric conductivity, respectively. We assume that $\mu, \sigma \in \mathrm{L}^{\infty}(\Omega)$, and that there exist constants $\underline{\mu}$ and $\underline{\sigma}$ such that

$$
\mu(\mathbf{x}) \geq \underline{\mu}>0 \quad \text { and } \quad \sigma(\mathbf{x}) \geq \underline{\sigma}>0, \quad \text { a.e. in } \Omega .
$$

The three-dimensional domain $\Omega$ is assumed to be simply connected with a Lipschitz-continuous and connected boundary $\partial \Omega$. This boundary splits into two surfaces of non-zero two-dimensional measure, $\Gamma_{E}$ and $\Gamma_{J}: \partial \Omega=\bar{\Gamma}_{E} \cup \bar{\Gamma}_{J}$. The (open) surface $\Gamma_{\mathrm{E}}$ corresponds to the tip of the electrode where the electric arc arises; we assume $\Gamma_{\mathrm{E}}$ is connected. The rest of the electrode boundary splits in its turn as follows: $\bar{\Gamma}_{\mathrm{J}}=\bar{\Gamma}_{\mathrm{J}}^{0} \cup \bar{\Gamma}_{\mathrm{J}}^{1} \cup \cdots \cup \bar{\Gamma}_{\mathrm{J}}^{N}$, where $\Gamma_{\mathrm{J}}^{n}, n=1, \ldots, N$, are the (open) parts of the boundary connected to the wires supplying electric current to the electrode, and $\Gamma_{\mathrm{J}}^{0}=\Gamma_{\mathrm{J}} \backslash\left(\bar{\Gamma}_{\mathrm{J}}^{1} \cup \cdots \cup \bar{\Gamma}_{\mathrm{J}}^{N}\right)$ is the remaining. Finally, we also assume that $\bar{\Gamma}_{\mathrm{J}}^{n} \cap \bar{\Gamma}_{\mathbf{E}}=\emptyset$ and $\bar{\Gamma}_{\mathrm{J}}^{n} \cap \bar{\Gamma}_{\mathrm{J}}^{m}=\emptyset, m, n=1, \ldots, N, m \neq n$ (see Figure 1 ).

Maxwell equations (2.1) -(2.3) concern the whole space, but we are only interested in a bounded conducting domain. To avoid dealing with the whole $\mathbb{R}^{3}$, it is necessary to use an alternative approach and define suitable boundary conditions. In fact, this need represents the main difficulty in solving the problem in a bounded domain. From a mathematical point of view, the essential and natural boundary conditions for the weak formulation written in terms of the magnetic field consist of giving 


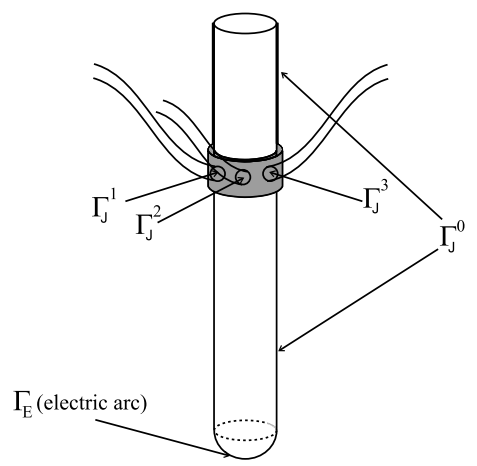

FiguRE 1. Sketch of an electrode

$\mathbf{H} \times \mathbf{n}$ and $\mathbf{E} \times \mathbf{n}$, respectively, $\mathbf{n}$ being the outward unit normal vector to the boundary. Both are easy to handle from mathematical and computational points of view. However they are not so easy to obtain from the physical data which usually reduces to the input current intensities. Then, following Bossavit [11, we consider the following boundary conditions:

$$
\begin{aligned}
\mathbf{E} \times \mathbf{n}=\mathbf{0} & \text { on } \Gamma_{\mathbf{E}}, \\
\int_{\Gamma_{\mathbf{J}}^{n}} \mathbf{c u r l} \mathbf{H} \cdot \mathbf{n}=I_{n}, & n=1, \ldots, N, \\
\mathbf{E} \times \mathbf{n}=\mathbf{0} & \text { on } \Gamma_{\mathbf{J}}^{n}, \quad n=1, \ldots, N, \\
\operatorname{curl} \mathbf{H} \cdot \mathbf{n}=0 & \text { on } \Gamma_{\mathbf{J}}^{0}, \\
\mu \mathbf{H} \cdot \mathbf{n}=0 & \text { on } \partial \Omega,
\end{aligned}
$$

where the only data $I_{n}, n=1, \ldots, N$, are the current intensities through each wire.

Condition (2.4) is the natural one to model the current free exit on the electrode tip. Conditions (2.5) and (2.7) account for the input intensities and the fact that there is no current flux through $\Gamma_{\mathrm{J}}^{0}$, respectively. Conditions (2.6) and (2.8) have been proposed by Bossavit in [11] in a more general setting. They will appear as natural boundary conditions of our weak formulation of the problem. The former implies the assumption that the electric current is normal to the surface on the current entrance, whereas the latter means that the magnetic field is tangential to the conductor surface. Of course, condition (2.8) is not always fulfilled, but is a good approximation of the physical one in the case motivating this study.

The model described above restricts the Maxwell equations to a conducting bounded domain and neglects the electromagnetic effects outside. To take into account this effect, one could employ, for instance, the three-dimensional model presented in [7]. However, as mentioned above, that model is much more expensive from a computational point of view.

As a first step toward the analysis of problem (2.1) -(2.8) we consider another related problem. It corresponds to the following boundary conditions:

$$
\begin{aligned}
\mathbf{E} \times \mathbf{n}=\mathbf{0} & \text { on } \Gamma_{\mathbf{E}}, \\
\operatorname{curl} \mathbf{H} \cdot \mathbf{n}=g & \text { on } \Gamma_{\mathrm{J}}, \\
\mu \mathbf{H} \cdot \mathbf{n}=0 & \text { on } \partial \Omega,
\end{aligned}
$$


where $g$ is a given function which is only non-zero on those parts of the boundary supporting input currents; i.e., $g=0$ on $\Gamma_{\mathrm{J}}^{0}$. Notice that $g$ corresponds to the input current density flux through $\Gamma_{\mathrm{J}}$, which is usually unknown on $\Gamma_{\mathrm{J}}^{n}$, for $n=1, \ldots, N$. Thus, this problem is less interesting from the practical point of view.

Throughout the paper all function spaces will be complex-valued. We use standard notation for Sobolev spaces and norms. Moreover, we recall the following definitions:

$$
\begin{aligned}
\mathrm{H}(\operatorname{div}, \Omega) & :=\left\{\mathbf{G} \in \mathrm{L}^{2}(\Omega)^{3}: \operatorname{div} \mathbf{G} \in \mathrm{L}^{2}(\Omega)\right\}, \\
\mathrm{H}(\operatorname{curl}, \Omega) & :=\left\{\mathbf{G} \in \mathrm{L}^{2}(\Omega)^{3}: \operatorname{curl} \mathbf{G} \in \mathrm{L}^{2}(\Omega)^{3}\right\},
\end{aligned}
$$

and, for each positive real number $r$,

$$
\mathrm{H}^{r}(\operatorname{curl}, \Omega):=\left\{\mathbf{G} \in \mathrm{H}^{r}(\Omega)^{3}: \operatorname{curl} \mathbf{G} \in \mathrm{H}^{r}(\Omega)^{3}\right\},
$$

each one of these spaces endowed with its natural norm, e.g., $\|\mathbf{G}\|_{\mathrm{H}^{r}(\mathbf{c u r l}, \Omega)}^{2}=$ $\|\mathbf{G}\|_{\mathrm{H}^{r}(\Omega)^{3}}^{2}+\|\operatorname{curl} \mathbf{G}\|_{\mathrm{H}^{r}(\Omega)^{3}}^{2}$.

Let $\mathrm{H}^{1 / 2}(\partial \Omega)$ be the space of traces of functions in $\mathrm{H}^{1}(\Omega), \mathrm{H}^{-1 / 2}(\partial \Omega)$ its dual space, and $\langle\cdot, \cdot\rangle_{\partial \Omega}$ the corresponding duality pairing. Let

$$
\mathrm{H}_{\Gamma_{\mathbf{E}}}^{1}(\Omega):=\left\{v \in \mathrm{H}^{1}(\Omega):\left.v\right|_{\Gamma_{\mathbf{E}}}=0\right\} .
$$

Let $\mathrm{H}_{00}^{1 / 2}\left(\Gamma_{\mathbf{J}}\right)$ be the space of functions defined on $\Gamma_{\mathbf{J}}$ that extended by 0 to $\partial \Omega \backslash \Gamma_{\mathbf{J}}$ belong to $\mathrm{H}^{1 / 2}(\partial \Omega)$. Notice that $\mathrm{H}_{00}^{1 / 2}\left(\Gamma_{\mathrm{J}}\right)$ can also be seen as the space of traces on $\Gamma_{\mathbf{J}}$ of functions in $\mathrm{H}_{\Gamma_{\mathbf{E}}}^{1}(\Omega)$. Let $\mathrm{H}_{00}^{-1 / 2}\left(\Gamma_{\mathbf{J}}\right)$ be the dual space of $\mathrm{H}_{00}^{1 / 2}\left(\Gamma_{\mathbf{J}}\right)$ and $\langle\cdot, \cdot\rangle_{\Gamma_{\mathbf{J}}}$ the corresponding duality pairing.

We end this section by proving the following lemma, which will be used several times in the sequel. Here and thereafter, $C$ denotes a generic constant, not necessarily the same at each occurrence.

Lemma 2.1. Given $q \in \mathrm{H}_{00}^{-1 / 2}\left(\Gamma_{\mathbf{J}}\right), \exists \mathbf{G} \in \mathrm{H}(\operatorname{curl}, \Omega) \cap \mathrm{H}(\operatorname{div}, \Omega)$ such that

$$
\begin{aligned}
& \operatorname{div} \mathbf{G}=0 \quad \text { in } \Omega, \\
& \mathbf{G} \cdot \mathbf{n}=0 \quad \text { on } \partial \Omega \text {, } \\
& \operatorname{curl} \mathbf{G} \cdot \mathbf{n}=q \quad \text { in } \mathrm{H}_{00}^{-1 / 2}\left(\Gamma_{\mathrm{J}}\right),
\end{aligned}
$$

and the following estimate holds true with a constant $C$ independent of $q$ :

$$
\|\mathbf{G}\|_{\mathrm{H}(\mathbf{c u r l}, \Omega)} \leq C\|q\|_{\mathrm{H}_{00}^{-1 / 2}\left(\Gamma_{\mathrm{J}}\right)} .
$$

Consequently, if $\Omega$ is a Lipschitz polyhedron, then $\exists r \in\left(\frac{1}{2}, 1\right]$ such that $\mathbf{G} \in$ $\mathrm{H}^{r}(\Omega)^{3}$ and

$$
\|\mathbf{G}\|_{\mathrm{H}^{r}(\Omega)^{3}} \leq C\|q\|_{\mathrm{H}_{00}^{-1 / 2}\left(\Gamma_{\mathbf{J}}\right)} .
$$

Furthermore, if $q \in \mathrm{L}^{2}\left(\Gamma_{\mathbf{J}}\right)$, then $\exists s>0$ such that $\operatorname{curl} \mathbf{G} \in \mathrm{H}^{s}(\Omega)^{3}$ and

$$
\|\operatorname{curl} \mathbf{G}\|_{\mathrm{H}^{s}(\Omega)^{3}} \leq C\|q\|_{\mathrm{L}^{2}\left(\Gamma_{\mathrm{J}}\right)} \text {. }
$$

In both estimates, the constants $C$ are also independent of $q$.

Proof. Let $u \in \mathrm{H}_{\Gamma_{\mathbf{E}}}^{1}(\Omega)$ be the unique solution of

$$
\int_{\Omega} \nabla u \cdot \nabla \bar{v}=\langle q, v\rangle_{\Gamma_{\mathbf{J}}} \quad \forall v \in \mathrm{H}_{\Gamma_{\mathbf{E}}}^{1}(\Omega) .
$$


Then,

$$
\Delta u=0 \quad \text { in } \Omega, \quad \frac{\partial u}{\partial \mathbf{n}}=q \quad \text { in } \mathrm{H}_{00}^{-1 / 2}\left(\Gamma_{\mathrm{J}}\right), \quad \text { and } \quad\|\nabla u\|_{\mathrm{L}^{2}(\Omega)} \leq C\|q\|_{\mathrm{H}_{00}^{-1 / 2}\left(\Gamma_{\mathrm{J}}\right)} .
$$

Since $\operatorname{div}(\nabla u)=0$ and $\partial \Omega$ is connected, we know from Theorem 3.12 of 4 t that there exists a vector field $\mathbf{G} \in \mathrm{H}(\mathbf{c u r l}, \Omega) \cap \mathrm{H}(\operatorname{div}, \Omega)$ satisfying (2.12) and (2.13) and such that $\nabla u=\operatorname{curl} \mathbf{G}$. Then (2.14) holds true. Moreover, Corollary 3.16 of the same reference [4] yields $\|\mathbf{G}\|_{\mathrm{H}(\mathbf{c u r l}, \Omega)} \leq C\|\nabla u\|_{\mathrm{L}^{2}(\Omega)}$ and, consequently, (2.15) holds true, too.

Now, if $\Omega$ is a Lipschitz polyhedron, estimate (2.16) is a consequence of Proposition 3.7 of [4]. Finally, if $q \in \mathrm{L}^{2}\left(\Gamma_{\mathrm{J}}\right)$, from a classical a priori estimate (see [13]), we know that curl $\mathbf{G}=\nabla u \in \mathrm{H}^{s}(\Omega)^{3}$ for some $s>0$ and (2.17) holds true.

\section{ANALYSIS OF THE EDDY CURRENT PROBLEM WITH INPUT CURRENT DENSITY FLUX AS BOUNDARY DATA}

In this section we consider problem (2.1) $-(2.3)$ with the boundary conditions (2.9) -(2.11). First we obtain a weak formulation of this problem in terms of the magnetic field.

Let us consider a smooth test function $\mathbf{G}$ such that $\operatorname{curl} \mathbf{G} \cdot \mathbf{n}=0$ on $\Gamma_{\mathbf{J}}$. From (2.2) we have

$$
\mathrm{i} \omega \int_{\Omega} \mu \mathbf{H} \cdot \overline{\mathbf{G}}+\int_{\Omega} \operatorname{curl} \mathbf{E} \cdot \overline{\mathbf{G}}=0 .
$$

By formal calculations, the boundary condition (2.11) implies that the tangential component of the electric field $\mathbf{E}$ is a gradient. Indeed, by integrating $\mathrm{i} \omega \mu \mathbf{H} \cdot \mathbf{n}$ on any surface $S$ contained in $\partial \Omega$ and using (2.11), (2.2), and Stokes' Theorem, we obtain

$$
0=\mathrm{i} \omega \int_{S} \mu \mathbf{H} \cdot \mathbf{n}=-\int_{S} \operatorname{curl} \mathbf{E} \cdot \mathbf{n}=-\int_{\partial S} \mathbf{E} \cdot \mathbf{t}=-\int_{\partial S} \mathbf{n} \times(\mathbf{E} \times \mathbf{n}) \cdot \mathbf{t},
$$

with t being a unit vector tangent to $\partial S$. Therefore, since $\partial \Omega$ is simply connected, we can assert that there exists a surface potential, defined up to a constant. More precisely, there exists a sufficiently smooth scalar function $\phi$ defined in $\Omega$, such that $\mathbf{E} \times \mathbf{n}=\nabla \phi \times \mathbf{n}$ on $\partial \Omega$. On the other hand, since $\Gamma_{\mathbf{E}}$ is connected, (2.9) implies that $\phi$ must be constant on $\Gamma_{\mathbf{E}}$; moreover, $\phi$ can be chosen null on $\Gamma_{\mathbf{E}}$. Then, we can transform the second term of (3.1) by using Green's formulas as follows:

$$
\begin{aligned}
\int_{\Omega} \operatorname{curl} \mathbf{E} \cdot \overline{\mathbf{G}}=\int_{\Omega} \mathbf{E} \cdot \operatorname{curl} \overline{\mathbf{G}}-\int_{\partial \Omega} \mathbf{E} \times \mathbf{n} \cdot \overline{\mathbf{G}} \\
=\int_{\Omega} \mathbf{E} \cdot \operatorname{curl} \overline{\mathbf{G}}-\int_{\partial \Omega} \nabla \phi \times \mathbf{n} \cdot \overline{\mathbf{G}}=\int_{\Omega} \mathbf{E} \cdot \operatorname{curl} \overline{\mathbf{G}}+\int_{\Omega} \operatorname{curl} \overline{\mathbf{G}} \cdot \nabla \phi \\
=\int_{\Omega} \mathbf{E} \cdot \operatorname{curl} \overline{\mathbf{G}}+\int_{\partial \Omega} \phi \operatorname{curl} \overline{\mathbf{G}} \cdot \mathbf{n}=\int_{\Omega} \mathbf{E} \cdot \operatorname{curl} \overline{\mathbf{G}},
\end{aligned}
$$

where we have used that $\operatorname{curl} \mathbf{G} \cdot \mathbf{n}=0$ on $\Gamma_{\mathrm{J}}$ and $\phi=0$ on $\Gamma_{\mathbf{E}}$ in the last equality.

Now, by substituting this expression in (3.1), we obtain,

$$
\mathrm{i} \omega \int_{\Omega} \mu \mathbf{H} \cdot \overline{\mathbf{G}}+\int_{\Omega} \mathbf{E} \cdot \operatorname{curl} \overline{\mathbf{G}}=0 .
$$


On the other hand, equations (2.1) and (2.3) lead to $\mathbf{E}=\frac{1}{\sigma} \mathbf{c u r l} \mathbf{H}$, which allows us to eliminate $\mathbf{E}$ in the above equation. Thus, we finally have that

$$
\mathrm{i} \omega \int_{\Omega} \mu \mathbf{H} \cdot \overline{\mathbf{G}}+\int_{\Omega} \frac{1}{\sigma} \operatorname{curl} \mathbf{H} \cdot \operatorname{curl} \overline{\mathbf{G}}=0
$$

for all $\mathbf{G} \in \mathrm{H}(\operatorname{curl}, \Omega)$ such that $\operatorname{curl} \mathbf{G} \cdot \mathbf{n}=0$ on $\Gamma_{\mathbf{J}}$.

In what follows we will obtain and analyze two different weak formulations, depending on the regularity of the boundary data $g$.

3.1. Analysis without further assumptions. We start considering boundary data $g=$ curl $\mathbf{H} \cdot \mathbf{n}$ belonging to $\mathrm{H}_{00}^{-1 / 2}\left(\Gamma_{\mathbf{J}}\right)$, which corresponds to the more general setting since curl $\mathbf{H} \in \mathrm{H}(\operatorname{div}, \Omega)$.

Let us denote for brevity $\mathcal{X}:=\mathrm{H}(\operatorname{curl}, \Omega)$, and consider the following linear manifold of $\mathcal{X}$

$$
\mathcal{V}(g):=\left\{\mathbf{G} \in \mathcal{X}: \operatorname{curl} \mathbf{G} \cdot \mathbf{n}=g \text { in } \mathrm{H}_{00}^{-1 / 2}\left(\Gamma_{\mathbf{J}}\right)\right\},
$$

with its associated subspace

$$
\mathcal{V}(0)=\left\{\mathbf{G} \in \mathcal{X}: \operatorname{curl} \mathbf{G} \cdot \mathbf{n}=0 \text { on } \Gamma_{\mathbf{J}}\right\} .
$$

Let $a: \mathcal{X} \times \mathcal{X} \longrightarrow \mathbb{C}$ be the sesquilinear continuous form defined by

$$
a(\mathbf{H}, \mathbf{G}):=\mathrm{i} \omega \int_{\Omega} \mu \mathbf{H} \cdot \overline{\mathbf{G}}+\int_{\Omega} \frac{1}{\sigma} \operatorname{curl} \mathbf{H} \cdot \operatorname{curl} \overline{\mathbf{G}} .
$$

This form is clearly $\mathcal{X}$-elliptic; namely, $\exists \alpha>0$ such that

$$
|a(\mathbf{G}, \mathbf{G})| \geq \alpha\|\mathbf{G}\|_{\mathcal{X}}^{2} \quad \forall \mathbf{G} \in \mathcal{X} .
$$

Now we introduce the following problem:

Problem 3.1. Given $g \in \mathrm{H}_{00}^{-1 / 2}\left(\Gamma_{\mathbf{J}}\right)$, find $\mathbf{H}_{g} \in \mathcal{V}(g)$ such that

$$
a\left(\mathbf{H}_{g}, \mathbf{G}\right)=0 \quad \forall \mathbf{G} \in \mathcal{V}(0) .
$$

The following existence result is easily deduced:

Theorem 3.2. Given $g \in \mathrm{H}_{00}^{-1 / 2}\left(\Gamma_{\mathrm{J}}\right)$, Problem 3.1] has a unique solution $\mathbf{H}_{g}$.

Proof. The result follows from the fact that $a$ is elliptic in $\mathcal{X}$, so in particular in $\mathcal{V}(0)$, and that $\mathcal{V}(g)$ is a non-empty closed linear manifold of $\mathcal{X}$, which in its turn is a consequence of Lemma 2.1 .

A mixed formulation of Problem 3.1 may be used to avoid dealing with functions that satisfy the constraints associated with $\mathcal{V}(g)$ and $\mathcal{V}(0)$. It consists of handling the boundary condition (2.10) in a weak sense by introducing a Lagrange multiplier defined on the boundary $\Gamma_{\mathrm{J}}$.

Let us denote $\mathcal{M}:=\mathrm{H}_{00}^{1 / 2}\left(\Gamma_{\mathrm{J}}\right)$ and $b$ the sesquilinear form defined in $\mathcal{X} \times \mathcal{M}$ by

$$
b(\mathbf{G}, \nu):=\langle\operatorname{curl} \mathbf{G} \cdot \mathbf{n}, \nu\rangle_{\Gamma_{\mathbf{J}}} .
$$

The mixed problem associated with Problem 3.1 is the following:

Problem 3.3. Given $g \in \mathrm{H}_{00}^{-1 / 2}\left(\Gamma_{\mathrm{J}}\right)$, find $\mathbf{H}_{g} \in \mathcal{X}$ and $\lambda_{g} \in \mathcal{M}$ such that

$$
\begin{aligned}
& a\left(\mathbf{H}_{g}, \mathbf{G}\right)+b\left(\overline{\mathbf{G}}, \lambda_{g}\right)=0 \quad \forall \mathbf{G} \in \mathcal{X}, \\
& b\left(\mathbf{H}_{g}, \nu\right)=\langle g, \nu\rangle_{\Gamma_{\mathbf{J}}} \quad \forall \nu \in \mathcal{M} .
\end{aligned}
$$


The classical Babuška-Brezzi theory can be applied to prove that this problem is well posed.

Theorem 3.4. Given $g \in \mathrm{H}_{00}^{-1 / 2}\left(\Gamma_{\mathrm{J}}\right)$, let $\mathbf{H}_{g} \in \mathcal{X}$ be the solution of Problem 3.1. Then, there exists a unique $\lambda_{g} \in \mathcal{M}$ such that $\left(\mathbf{H}_{g}, \lambda_{g}\right)$ is the only solution of Problem 3.3. Furthermore, the following continuous dependence result holds:

$$
\left\|\mathbf{H}_{g}\right\|_{\mathcal{X}}+\left\|\lambda_{g}\right\|_{\mathcal{M}} \leq C\|g\|_{\mathrm{H}_{00}^{-1 / 2}\left(\Gamma_{\mathbf{J}}\right)} .
$$

Proof. Since the forms $a$ and $b$ are clearly continuous on their respective domains and $a$ is $\mathcal{X}$-elliptic, we only have to prove that $b$ satisfies an inf-sup condition (see for instance Corollary I.4.1 of [14]). More precisely, we are going to prove that there exists $\beta>0$ such that

$$
\sup _{\mathbf{G} \in \mathcal{X}} \frac{|b(\mathbf{G}, \nu)|}{\|\mathbf{G}\|_{\mathcal{X}}} \geq \beta\|\nu\|_{\mathcal{M}} \quad \forall \nu \in \mathcal{M} .
$$

Since $\mathcal{M}=\mathrm{H}_{00}^{1 / 2}\left(\Gamma_{\mathrm{J}}\right)$ is a Hilbert space, $\forall \nu \in \mathcal{M}$ we can write

$$
\|\nu\|_{\mathcal{M}}=\sup _{q \in \mathrm{H}_{00}^{-1 / 2}\left(\Gamma_{\mathbf{J}}\right)} \frac{\left|\langle q, \nu\rangle_{\Gamma_{\mathbf{J}}}\right|}{\|q\|_{\mathrm{H}_{00}^{-1 / 2}\left(\Gamma_{\mathbf{J}}\right)}} .
$$

According to Lemma 2.1 $\forall q \in \mathrm{H}_{00}^{-1 / 2}\left(\Gamma_{\mathbf{J}}\right), \exists \widetilde{\mathbf{G}} \in \mathcal{X}$ such that $\operatorname{curl} \widetilde{\mathbf{G}} \cdot \mathbf{n}=q$ in $\mathrm{H}_{00}^{-1 / 2}\left(\Gamma_{\mathbf{J}}\right)$ and $\|\widetilde{\mathbf{G}}\|_{\mathcal{X}} \leq C\|q\|_{\mathrm{H}_{00}^{-1 / 2}\left(\Gamma_{\mathbf{J}}\right)}$. Then

$$
\frac{\left|\langle q, \nu\rangle_{\Gamma_{\mathbf{J}}}\right|}{\|q\|_{\mathrm{H}_{00}^{-1 / 2}\left(\Gamma_{\mathbf{J}}\right)}} \leq C \frac{\left|\langle\operatorname{curl} \widetilde{\mathbf{G}} \cdot \mathbf{n}, \nu\rangle_{\Gamma_{\mathbf{J}}}\right|}{\|\widetilde{\mathbf{G}}\|_{\mathcal{X}}} \leq C \sup _{\mathbf{G} \in \mathcal{X}} \frac{\left|\langle\operatorname{curl} \mathbf{G} \cdot \mathbf{n}, \nu\rangle_{\Gamma_{\mathbf{J}}}\right|}{\|\mathbf{G}\|_{\mathcal{X}}} .
$$

Thus, the inf-sup condition holds with $\beta=\frac{1}{C}$.

Notice that once the magnetic field $\mathbf{H}_{g}$ is obtained, the corresponding current density $\mathbf{J}_{g}$ and electric field $\mathbf{E}_{g}$ can be readily computed in the domain $\Omega$ by means of equations (2.1) and (2.3), respectively. These are the magnitudes actually needed in most applications. In the following theorem we show that the solution of Problem 3.3 satisfies the Maxwell equations (2.1)-2.3) and the boundary conditions (2.9)(2.11) in a suitable sense.

Theorem 3.5. Given $g \in \mathrm{H}_{00}^{-1 / 2}\left(\Gamma_{\mathrm{J}}\right)$, let $\mathbf{H}_{g} \in \mathcal{X}$ and $\lambda_{g} \in \mathcal{M}$ be the solution of Problem 3.3 Let $\lambda_{g}^{*}$ be a lifting of $\lambda_{g}$ to $\Omega$ such that $\lambda_{g}^{*} \in \mathrm{H}_{\Gamma_{\mathbf{E}}}^{1}(\Omega)$. Let $\mathbf{J}_{g}:=$ $\operatorname{curl} \mathbf{H}_{g} \in \mathrm{L}^{2}(\Omega)^{3}$ and $\mathbf{E}_{g}:=\frac{1}{\sigma} \mathbf{J}_{g} \in \mathrm{L}^{2}(\Omega)^{3}$. Then, the following equalities hold true:

$$
\begin{aligned}
\mathrm{i} \omega \mu \mathbf{H}_{g}+\operatorname{curl} \mathbf{E}_{g}=\mathbf{0} & \text { in } \Omega, \\
\mu \mathbf{H}_{g} \cdot \mathbf{n}=0 & \text { on } \partial \Omega, \\
\operatorname{curl} \mathbf{H}_{g} \cdot \mathbf{n}=g & \text { in } \mathrm{H}_{00}^{-1 / 2}\left(\Gamma_{\mathbf{J}}\right) . \\
\mathbf{E}_{g} \times \mathbf{n}=-\nabla \bar{\lambda}_{g}^{*} \times \mathbf{n} & \text { in } \mathrm{H}^{-1 / 2}(\partial \Omega)^{3} .
\end{aligned}
$$

In particular,

$$
\mathbf{E}_{g} \times \mathbf{n}=\mathbf{0} \quad \text { on } \Gamma_{\mathbf{E}}
$$


Proof. Let $\mathbf{G} \in \mathcal{D}(\Omega)^{3}$. Then $\mathbf{G} \in \mathcal{X}$ and (3.3) yields

$$
\mathrm{i} \omega \int_{\Omega} \mu \mathbf{H}_{g} \cdot \overline{\mathbf{G}}+\int_{\Omega} \frac{1}{\sigma} \operatorname{curl} \mathbf{H}_{g} \cdot \operatorname{curl} \overline{\mathbf{G}}=0 .
$$

Hence $\mathbf{E}_{g}=\frac{1}{\sigma} \mathbf{J}_{g}=\frac{1}{\sigma} \operatorname{curl} \mathbf{H}_{g} \in \mathrm{H}(\operatorname{curl}, \Omega)$ and (3.6) holds true.

Condition (3.8) is directly deduced from (3.4), where this boundary condition is imposed in a variational form.

To prove equation (3.9), given $\phi \in \mathrm{H}^{1 / 2}(\partial \Omega)^{3}$, we are going to show that $\left\langle\mathbf{E}_{g} \times \mathbf{n}, \boldsymbol{\phi}\right\rangle_{\partial \Omega}=-\left\langle\nabla \bar{\lambda}_{g}^{*} \times \mathbf{n}, \boldsymbol{\phi}\right\rangle_{\partial \Omega}$. To this aim, let $\mathbf{G} \in \mathrm{H}^{1}(\Omega)^{3}$ be such that $\left.\mathbf{G}\right|_{\partial \Omega}=\boldsymbol{\phi}$. Then $\mathbf{G} \in \mathcal{X}$ and (3.3) yields

$$
\begin{aligned}
0 & =\mathrm{i} \omega \int_{\Omega} \mu \mathbf{H}_{g} \cdot \overline{\mathbf{G}}+\int_{\Omega} \mathbf{E}_{g} \cdot \operatorname{curl} \overline{\mathbf{G}}+\left\langle\operatorname{curl} \overline{\mathbf{G}} \cdot \mathbf{n}, \lambda_{g}\right\rangle_{\Gamma_{\mathbf{J}}} \\
& =\mathrm{i} \omega \int_{\Omega} \mu \mathbf{H}_{g} \cdot \overline{\mathbf{G}}+\int_{\Omega} \mathbf{E}_{g} \cdot \operatorname{curl} \overline{\mathbf{G}}+\left\langle\operatorname{curl} \overline{\mathbf{G}} \cdot \mathbf{n},\left.\lambda_{g}^{*}\right|_{\partial \Omega}\right\rangle_{\partial \Omega} \\
& =\mathrm{i} \omega \int_{\Omega} \mu \mathbf{H}_{g} \cdot \overline{\mathbf{G}}+\int_{\Omega} \operatorname{curl} \mathbf{E}_{g} \cdot \overline{\mathbf{G}}+\left\langle\mathbf{E}_{g} \times \mathbf{n},\left.\mathbf{G}\right|_{\partial \Omega}\right\rangle_{\partial \Omega}+\int_{\Omega} \operatorname{curl} \overline{\mathbf{G}} \cdot \nabla \bar{\lambda}_{g}^{*} \\
& =\left\langle\mathbf{E}_{g} \times \mathbf{n}, \boldsymbol{\phi}\right\rangle_{\partial \Omega}+\left\langle\nabla \bar{\lambda}_{g}^{*} \times \mathbf{n}, \boldsymbol{\phi}\right\rangle_{\partial \Omega},
\end{aligned}
$$

where we have used that $\mathbf{E}_{g}=\frac{1}{\sigma} \operatorname{curl} \mathbf{H}_{g}$ in $\Omega$, Green's formulas, and (3.6).

Finally, to prove (3.7), notice that $\mu \mathbf{H}_{g} \in \mathrm{H}(\operatorname{div}, \Omega)$ because of (3.6). Then, $\mu \mathbf{H}_{g} \cdot \mathbf{n} \in \mathrm{H}^{-1 / 2}(\partial \Omega)$ and given $\psi \in \mathcal{C}^{\infty}(\bar{\Omega})$ we have

$$
\begin{aligned}
\mathrm{i} \omega\left\langle\mu \mathbf{H}_{g} \cdot \mathbf{n}, \psi\right\rangle_{\partial \Omega} & =-\left\langle\operatorname{curl}_{g} \cdot \mathbf{n}, \psi\right\rangle_{\partial \Omega}=-\int_{\Omega} \operatorname{curl} \mathbf{E}_{g} \cdot \nabla \bar{\psi} \\
& =-\left\langle\nabla \bar{\lambda}_{g}^{*} \times \mathbf{n}, \nabla \psi\right\rangle_{\partial \Omega}=-\int_{\Omega} \operatorname{curl}\left(\nabla \bar{\lambda}_{g}^{*}\right) \cdot \nabla \bar{\psi}=0,
\end{aligned}
$$

where we have used (3.6), (3.9), and Green's formulas.

The following direct consequence of this theorem, which will be used several times in Section 5 below, shows that a smoothness assumption on the magnetic field $\mathbf{H}_{g}$ implies further smoothness of the Lagrange multiplier $\lambda_{g}$ too.

Corollary 3.6. Let $\Gamma$ be a smooth piece of $\Gamma_{\mathrm{J}}$ such that $\left.\sigma\right|_{\Gamma}$ is also smooth (e.g., $\left.\mathcal{C}^{2}\right)$. If $\mathbf{H}_{g} \in \mathrm{H}^{r}(\operatorname{curl}, \Omega)$ with $1 / 2<r \leq 1$, then $\left.\lambda_{g}\right|_{\Gamma} \in \mathrm{H}^{r+1 / 2}(\Gamma)$ and

$$
\left\|\lambda_{g}\right\|_{\mathrm{H}^{r+1 / 2}(\Gamma)} \leq C\left\|\mathbf{H}_{g}\right\|_{\mathrm{H}^{r}(\mathbf{c u r l}, \Omega)} .
$$

Proof. As shown in the proof of Theorem $3.4 b$ satisfies the inf-sup condition (3.5). Then, from equation (3.3) and the continuity of $a$ we have that $\left\|\lambda_{g}\right\|_{\mathcal{M}} \leq$ $\left(\|\alpha\|_{\mathcal{X} \times \mathcal{X}} / \beta\right)\left\|\mathbf{H}_{g}\right\|_{\mathcal{X}}$. Hence $\left\|\lambda_{g}\right\|_{\mathrm{L}^{2}\left(\Gamma_{\mathrm{J}}\right)} \leq C\left\|\mathbf{H}_{g}\right\|_{\mathrm{H}(\operatorname{curl}, \Omega)}$.

On the other hand, since $\operatorname{curl} \mathbf{H}_{g} \in \mathrm{H}^{r}(\Omega)^{3}$, because of the standard trace theorem, $\left.\operatorname{curl} \mathbf{H}_{g}\right|_{\Gamma} \in \mathrm{H}^{r-1 / 2}(\Gamma)^{3}$. Then, since $\Gamma$ and $\left.\sigma\right|_{\Gamma} \geq \underline{\sigma}>0$ are assumed to be smooth, we have

$$
-\left.\nabla \bar{\lambda}_{g}^{*}\right|_{\Gamma} \times \mathbf{n}=\left.\mathbf{E}_{g}\right|_{\Gamma} \times \mathbf{n}=\left.\frac{1}{\sigma} \operatorname{curl} \mathbf{H}_{g}\right|_{\Gamma} \times \mathbf{n} \in \mathrm{H}^{r-1 / 2}(\Gamma)^{3} .
$$

Hence, the surface gradient of $\lambda_{g}$ on $\Gamma, \nabla_{\Gamma} \lambda_{g}:=\mathbf{n} \times\left(\left.\nabla \bar{\lambda}_{g}^{*}\right|_{\Gamma} \times \mathbf{n}\right)$, satisfies $\nabla_{\Gamma} \lambda_{g} \in$ $\mathrm{H}^{r-1 / 2}(\Gamma)^{3}$ too, and $\left\|\nabla_{\Gamma} \lambda_{g}\right\|_{\mathrm{H}^{r-1 / 2}(\Gamma)} \leq C\left\|\mathbf{H}_{g}\right\|_{\mathrm{H}^{r}(\mathbf{c u r l}, \Omega)}$. Thus, we conclude the proof. 
Problem 3.3 can be discretized by using Nédélec edge finite elements for the magnetic field $\mathbf{H}_{g}$ and piecewise linear continuous elements for the Lagrange multiplier $\lambda_{g}$. The resulting discrete problem and its convergence properties are studied in detail in Section 5.1

However, at this point, it is important to remark that if the boundary data $g$ were more regular, it would be possible to use piecewise constant functions to approximate the Lagrange multiplier $\lambda_{g}$. For instance, this is true if $g \in \mathrm{L}^{2}\left(\Gamma_{\mathrm{J}}\right)$, which is quite a realistic assumption. Before studying this possibility at a discrete level, we deal with the corresponding continuous problem in what follows.

3.2. Analysis for input current density flux in $\mathrm{L}^{2}\left(\Gamma_{\mathrm{J}}\right)$. In this section we study problem (2.1) - 2.3) with the boundary conditions 2.9 -2.11) in the case that the boundary data $g \in \mathrm{L}^{2}\left(\Gamma_{\mathrm{J}}\right)$. To this goal we follow a scheme similar to that of the previous section.

Let $\widetilde{\mathcal{X}}$ be the subspace of $\mathcal{X}$ defined by

$$
\widetilde{\mathcal{X}}:=\left\{\mathbf{G} \in \mathrm{H}(\operatorname{curl}, \Omega):\left.\operatorname{curl} \mathbf{G} \cdot \mathbf{n}\right|_{\Gamma_{\mathbf{J}}} \in \mathrm{L}^{2}\left(\Gamma_{\mathbf{J}}\right)\right\},
$$

which is a Hilbert space when equipped with the norm

$$
\|\mathbf{G}\|_{\widetilde{\mathcal{X}}}:=\left[\|\mathbf{G}\|_{\mathrm{H}(\operatorname{curl}, \Omega)}^{2}+\|\operatorname{curl} \mathbf{G} \cdot \mathbf{n}\|_{\mathrm{L}^{2}\left(\Gamma_{\mathbf{J}}\right)}^{2}\right]^{1 / 2} .
$$

Consider the following linear manifold of $\widetilde{\mathcal{X}}$ :

$$
\widetilde{\mathcal{V}}(g):=\left\{\mathbf{G} \in \widetilde{\mathcal{X}}: \operatorname{curl} \mathbf{G} \cdot \mathbf{n}=g \text { on } \Gamma_{\mathbf{J}}\right\},
$$

and its associated subspace $\widetilde{\mathcal{V}}(0)$, which coincides with $\mathcal{V}(0)$. We introduce the following problem:

Problem 3.7. Given $g \in \mathrm{L}^{2}\left(\Gamma_{\mathbf{J}}\right)$, find $\mathbf{H}_{g} \in \widetilde{\mathcal{V}}(g)$ such that

$$
a\left(\mathbf{H}_{g}, \mathbf{G}\right)=0 \quad \forall \mathbf{G} \in \widetilde{\mathcal{V}}(0) .
$$

An existence result for Problem 3.7 is easily deduced:

Theorem 3.8. Given $g \in \mathrm{L}^{2}\left(\Gamma_{\mathrm{J}}\right)$, Problem 3.7 has a unique solution $\mathbf{H}_{g}$.

Proof. The result follows from the fact that $a$ is $\widetilde{\mathcal{X}}$-elliptic in $\tilde{\mathcal{V}}(0)$ and that $\tilde{\mathcal{V}}(g)$ is a non-empty closed linear manifold of $\mathrm{H}(\operatorname{curl}, \Omega)$, which is again a consequence of Lemma 2.1.

We consider again a mixed problem to handle the constraints involved in $\tilde{\mathcal{V}}(g)$ and $\widetilde{\mathcal{V}}(0)$. Let $\widetilde{\mathcal{M}}:=\mathrm{L}^{2}\left(\Gamma_{\mathbf{J}}\right)$. Notice that if $(\mathbf{G}, \nu) \in \widetilde{\mathcal{X}} \times \widetilde{\mathcal{M}}$, then

$$
b(\mathbf{G}, \nu)=\int_{\Gamma_{\mathbf{J}}} \operatorname{curl} \mathbf{G} \cdot \mathbf{n} \bar{\nu} .
$$

The mixed problem associated with Problem 3.7 reads as follows:

Problem 3.9. Given $g \in \mathrm{L}^{2}\left(\Gamma_{\mathrm{J}}\right)$, find $\mathbf{H}_{g} \in \widetilde{\mathcal{X}}$ and $\lambda_{g} \in \widetilde{\mathcal{M}}$ such that

$$
\begin{aligned}
& a\left(\mathbf{H}_{g}, \mathbf{G}\right)+b\left(\overline{\mathbf{G}}, \lambda_{g}\right)=0 \quad \forall \mathbf{G} \in \widetilde{\mathcal{X}}, \\
& b\left(\mathbf{H}_{g}, \nu\right)=\int_{\Gamma_{\mathbf{J}}} g \bar{\nu} \quad \forall \nu \in \widetilde{\mathcal{M}} .
\end{aligned}
$$


We apply again the classical Babuška-Brezzi theory to prove that this problem is well posed.

Theorem 3.10. Given $g \in \mathrm{L}^{2}\left(\Gamma_{\mathrm{J}}\right)$, let $\mathbf{H}_{g} \in \widetilde{\mathcal{X}}$ be the solution of Problem 3.7. Then there exists a unique $\lambda_{g} \in \widetilde{\mathcal{M}}$ such that $\left(\mathbf{H}_{g}, \lambda_{g}\right)$ is the only solution of Problem [3.9. Furthermore, the following continuous dependence result holds:

$$
\left\|\mathbf{H}_{g}\right\|_{\widetilde{\mathcal{X}}}+\left\|\lambda_{g}\right\|_{\widetilde{\mathcal{M}}} \leq C\|g\|_{\mathrm{L}^{2}\left(\Gamma_{\mathrm{J}}\right)} \text {. }
$$

Proof. Since $a$ and $b$ are clearly continuous on their respective domains and $a$ is $\widetilde{\mathcal{X}}$ elliptic in $\widetilde{\mathcal{V}}(0)$, we only have to prove that $b$ satisfies the following inf-sup condition (see for instance Corollary I.4.1 of [14] again):

$$
\sup _{\mathbf{G} \in \widetilde{\mathcal{X}}} \frac{|b(\mathbf{G}, \nu)|}{\|\mathbf{G}\|_{\widetilde{\mathcal{X}}}} \geq \widetilde{\beta}\|\nu\|_{\widetilde{\mathcal{M}}} \quad \forall \nu \in \widetilde{\mathcal{M}}
$$

To prove this, given $\nu \in \widetilde{\mathcal{M}}$, let $\widetilde{\mathbf{G}} \in \mathcal{X}$ be the vector field associated with $q=\nu$ by Lemma 2.1. Because of (2.14), $\widetilde{\mathbf{G}} \in \widetilde{\mathcal{X}}$; moreover, $\|\widetilde{\mathbf{G}}\|_{\widetilde{\mathcal{X}}} \leq C\|\nu\|_{\widetilde{\mathcal{M}}}$ because of (2.15). Hence,

$$
\sup _{\mathbf{G} \in \widetilde{\mathcal{X}}} \frac{|b(\mathbf{G}, \nu)|}{\|\mathbf{G}\|_{\widetilde{\mathcal{X}}}} \geq \frac{\left|\int_{\Gamma_{\mathbf{J}}} \operatorname{curl} \widetilde{\mathbf{G}} \cdot \mathbf{n} \bar{\nu}\right|}{\|\widetilde{\mathbf{G}}\|_{\widetilde{\mathcal{X}}}} \geq \frac{\int_{\Gamma_{\mathbf{J}}}|\nu|^{2}}{C\|\nu\|_{L^{2}\left(\Gamma_{\mathbf{J}}\right)}}=\frac{1}{C}\|\nu\|_{L^{2}\left(\Gamma_{\mathbf{J}}\right)},
$$

which allows us to conclude the proof by taking $\widetilde{\beta}=\frac{1}{C}$.

Remark 3.11. If $g \in \mathrm{L}^{2}\left(\Gamma_{\mathrm{J}}\right)$, the solution of Problem[3.3 is also a solution of Problem 3.9, because $\mathcal{M}$ is dense in $\widetilde{\mathcal{M}}$. This is the reason why we use the same notation for the solutions of both problems. So we could deduce the existence of the solution of Problem 3.9 from Theorem 3.4 However this is not the case for uniqueness and this is the reason why we have proved Theorem 3.10 Consequently, the solution of Problem 3.9 also satisfies Theorem 3.5 and Corollary 3.6

\section{Analysis of the EDdy CURRENT PRoblem WITH INPUT CURRENT INTENSITIES AS BOUNDARY DATA}

We address now our main goal: to solve the eddy current model with the boundary conditions (2.4) -(2.8). We introduce weak formulations related to these boundary conditions and use the results obtained in the previous section for Problems 3.3 and 3.9 as tools for our analysis.

We study again two different cases according to the regularity of the corresponding current density flux through $\Gamma_{J}$.

4.1. Analysis without further assumptions. Given (complex) input intensities $I_{1}, \ldots, I_{N}$ through each wire, let us denote $\mathbf{I}:=\left(I_{1}, \ldots, I_{N}\right) \in \mathbb{C}^{N}$ and $I \in \mathrm{L}^{2}\left(\Gamma_{\mathbf{J}}\right)$ the function defined by

$$
I:= \begin{cases}\frac{I_{n}}{\operatorname{meas}\left(\Gamma_{\mathrm{J}}^{n}\right)} & \text { on } \Gamma_{\mathrm{J}}^{n}, \quad n=1, \ldots, N, \\ 0 & \text { on } \Gamma_{\mathrm{J}}^{0} .\end{cases}
$$

Let $\mathcal{L}$ be the following closed subspace of $\mathcal{M}$ (recall that $\mathcal{M}=\mathrm{H}_{00}^{1 / 2}\left(\Gamma_{\mathrm{J}}\right)$ ):

$$
\mathcal{L}:=\left\{\nu \in \mathrm{H}_{00}^{1 / 2}\left(\Gamma_{\mathrm{J}}\right):\left.\nu\right|_{\Gamma_{\mathrm{J}}^{n}}=\text { constant, } n=1, \ldots, N\right\} .
$$


Consider the following closed linear manifold of $\mathcal{X}$,

$$
\mathcal{W}(I):=\left\{\mathbf{G} \in \mathcal{X}:\langle\operatorname{curl} \mathbf{G} \cdot \mathbf{n}, \nu\rangle_{\Gamma_{\mathbf{J}}}=\int_{\Gamma_{\mathbf{J}}} I \bar{\nu} \quad \forall \nu \in \mathcal{L}\right\},
$$

and its associated subspace

$$
\mathcal{W}(0)=\left\{\mathbf{G} \in \mathcal{X}:\langle\operatorname{curl} \mathbf{G} \cdot \mathbf{n}, \nu\rangle_{\Gamma_{\mathbf{J}}}=0 \quad \forall \nu \in \mathcal{L}\right\} .
$$

We introduce the following problem:

Problem 4.1. Given $\mathbf{I} \in \mathbb{C}^{N}$, find $\mathbf{H}_{\mathbf{I}} \in \mathcal{W}(I)$ such that

$$
a\left(\mathbf{H}_{\mathbf{I}}, \mathbf{G}\right)=0 \quad \forall \mathbf{G} \in \mathcal{W}(0) .
$$

Theorem 4.2. Given $\mathbf{I} \in \mathbb{C}^{N}$, Problem 4.1 has a unique solution $\mathbf{H}_{\mathbf{I}}$.

Proof. The result is an immediate consequence of the fact that $a$ is $\mathcal{X}$-elliptic and $\mathcal{W}(I)$ is a non-empty closed linear manifold. Notice that $\mathcal{W}(I)$ is non-empty because $\mathcal{W}(I) \supset \mathcal{V}(I)$, and we have already proved that $\mathcal{V}(g)$ is non-empty $\forall g \in$ $\mathrm{L}^{2}\left(\Gamma_{\mathrm{J}}\right)$.

We consider a mixed problem for handling the constraints in $\mathcal{W}(I)$ and $\mathcal{W}(0)$ in a way similar to how we did in the previous sections:

Problem 4.3. Given $\mathbf{I} \in \mathbb{C}^{N}$, find $\mathbf{H}_{\mathbf{I}} \in \mathcal{X}$ and $\lambda_{\mathbf{I}} \in \mathcal{L}$ such that

$$
\begin{aligned}
& a\left(\mathbf{H}_{\mathbf{I}}, \mathbf{G}\right)+b\left(\overline{\mathbf{G}}, \lambda_{\mathbf{I}}\right)=0 \quad \forall \mathbf{G} \in \mathcal{X}, \\
& b\left(\mathbf{H}_{\mathbf{I}}, \nu\right)=\int_{\Gamma_{\mathbf{J}}} I \bar{\nu} \quad \forall \nu \in \mathcal{L} .
\end{aligned}
$$

Theorem 4.4. Given $\mathbf{I} \in \mathbb{C}^{N}$, let $\mathbf{H}_{\mathbf{I}} \in \mathcal{X}$ be the solution of Problem 4.1. Then there exists a unique $\lambda_{\mathbf{I}} \in \mathcal{L}$ such that $\left(\mathbf{H}_{\mathbf{I}}, \lambda_{\mathbf{I}}\right)$ is the only solution of Problem 4.3 . Furthermore, the following estimate holds:

$$
\left\|\mathbf{H}_{\mathbf{I}}\right\|_{\mathcal{X}}+\left\|\lambda_{\mathbf{I}}\right\|_{\mathcal{M}} \leq C|\mathbf{I}|
$$

Proof. The existence and uniqueness results are immediate consequences of the facts that $a$ is $\mathcal{X}$-elliptic and that the corresponding inf-sup condition for $b$ holds true in $\mathcal{M}$ (and a fortiori in $\mathcal{L} \subset \mathcal{M}$ ) as shown in the proof of Theorem 3.4 (see for instance Corollary I.4.1 of 14 once more). Moreover,

$$
\left\|\mathbf{H}_{\mathbf{I}}\right\|_{\mathcal{X}}+\left\|\lambda_{\mathbf{I}}\right\|_{\mathcal{M}} \leq C \sup _{\nu \in \mathcal{L}} \frac{\left|\int_{\Gamma_{\mathbf{J}}} I \bar{\nu}\right|}{\|\nu\|_{\mathcal{M}}} \leq C\|I\|_{\mathrm{L}^{2}\left(\Gamma_{\mathbf{J}}\right)} \leq C|\mathbf{I}| .
$$

Thus, we conclude the proof.

Remark 4.5. The arguments in the proof of Theorem 3.5 apply to the solution $\mathbf{H}_{\mathbf{I}} \in \mathcal{X}$ and $\lambda_{\mathbf{I}} \in \mathcal{L}$ of Problem 4.3, yielding equations analogous to (3.6)-(3.10), except for (3.8). Instead of this, $\mathbf{H}_{\mathbf{I}}$ satisfies in this case $\left\langle\mathbf{c u r l} \mathbf{H}_{\mathbf{I}} \cdot \mathbf{n}, \nu\right\rangle_{\Gamma_{\mathbf{J}}}=\int_{\Gamma_{\mathbf{J}}} I \bar{\nu}$ $\forall \nu \in \mathcal{L}$. This equation implies the boundary condition (2.7):

$$
\operatorname{curl} \mathbf{H}_{\mathbf{I}} \cdot \mathbf{n}=0 \quad \text { on } \Gamma_{\mathbf{J}}^{0} \text {. }
$$

Moreover, for $n=1, \ldots, N,\left\langle\operatorname{curl} \mathbf{H}_{\mathbf{I}} \cdot \mathbf{n}, 1\right\rangle_{\Gamma_{\mathbf{J}}^{n}}$ is well defined. Indeed, let $\nu_{n} \in \mathcal{L}$ be any function such that $\nu_{n}=1$ on $\Gamma_{\mathbf{J}}^{n}$ and $\nu_{n}=0$ on $\Gamma_{\mathbf{J}}^{m}$ for $m \neq n$. (Notice that such $\nu_{n}$ exists because of the assumption $\bar{\Gamma}_{\mathbf{J}}^{n} \cap \bar{\Gamma}_{\mathbf{J}}^{m}=\emptyset, m \neq n$.) Then, 
$\left\langle\operatorname{curl} \mathbf{H}_{\mathbf{I}} \cdot \mathbf{n}, 1\right\rangle_{\Gamma_{\mathbf{J}}^{n}}:=\left\langle\operatorname{curl}_{\mathbf{H}} \mathbf{H}_{\mathbf{I}} \cdot \mathbf{n}, \nu_{n}\right\rangle_{\Gamma_{\mathbf{J}}}$ is well defined, since the latter does not depend on the particular chosen function $\nu_{n}$, because of (2.7). Furthermore, (4.2) leads to the following form of the boundary condition (2.5):

$$
\left\langle\operatorname{curl} \mathbf{H}_{\mathbf{I}} \cdot \mathbf{n}, 1\right\rangle_{\Gamma_{\mathbf{J}}^{n}}=I_{n}, \quad n=1, \ldots, N .
$$

On the other hand, taking into account that the Lagrange multiplier $\lambda_{\mathbf{I}}$ is constant on each $\Gamma_{\mathrm{J}}^{n}, n=1, \ldots, N$, we deduce from (3.9) that $\mathbf{E}_{\mathbf{I}}=\frac{1}{\sigma} \mathbf{c u r l} \mathbf{H}_{\mathbf{I}}$ satisfies the boundary condition (2.6):

$$
\mathbf{E}_{\mathbf{I}} \times \mathbf{n}=\mathbf{0} \quad \text { on } \Gamma_{\mathrm{J}}^{n}, \quad n=1, \ldots, N .
$$

Finally, the analogue to Corollary [3.6] also holds true: If $\Gamma \subset \partial \Omega,\left.\sigma\right|_{\Gamma}$ is smooth, and $\mathbf{H}_{\mathbf{I}} \in \mathrm{H}^{r}(\operatorname{curl}, \Omega)$ with $1 / 2<r \leq 1$, then $\left.\lambda_{\mathbf{I}}\right|_{\Gamma} \in \mathrm{H}^{r+1 / 2}(\Gamma)$ and

$$
\left\|\lambda_{\mathbf{I}}\right\|_{\mathrm{H}^{r+1 / 2}(\Gamma)} \leq C\left\|\mathbf{H}_{\mathbf{I}}\right\|_{\mathrm{H}^{r}(\mathbf{c u r l}, \Omega)} .
$$

Problem 4.3 can be discretized by using Nédélec edge finite elements for the magnetic field $\mathbf{H}_{\mathbf{I}}$ and piecewise linear continuous elements which are constant on $\Gamma_{\mathrm{J}}^{n}$, $n=1, \ldots, N$, for the Lagrange multiplier $\lambda_{\mathbf{I}}$. In a way similar to how we noticed for Problem 3.3, if the (unknown) input current density flux through $\Gamma_{\mathrm{J}}$ satisfies $\operatorname{curl} \mathbf{H}_{\mathbf{I}} \cdot \mathbf{n} \in \mathrm{L}^{2}\left(\Gamma_{\mathbf{J}}\right)$, then we can employ piecewise constant functions to approximate the Lagrange multiplier $\lambda_{\mathbf{I}}$. In what follows we present the corresponding continuous problem in order to study later its discrete approximation.

4.2. Analysis for input current density flux in $L^{2}\left(\Gamma_{J}\right)$. In this section we study the eddy current model with the boundary conditions (2.4)-(2.8) in the case that the input current density flux through $\Gamma_{\mathrm{J}}$ is an $\mathrm{L}^{2}$-function. This amounts to assuming a bit more of regularity of $\mathbf{H}_{\mathbf{I}}$, namely, that $\operatorname{curl} \mathbf{H}_{\mathbf{I}} \cdot \mathbf{n} \in \mathrm{L}^{2}\left(\Gamma_{\mathbf{J}}\right)$.

Let $\widetilde{\mathcal{L}}$ be the following closed subspace of $\widetilde{\mathcal{M}}$ (recall that $\widetilde{\mathcal{M}}=\mathrm{L}^{2}\left(\Gamma_{\mathrm{J}}\right)$ ):

$$
\widetilde{\mathcal{L}}:=\left\{\nu \in \mathrm{L}^{2}\left(\Gamma_{\mathrm{J}}\right):\left.\nu\right|_{\Gamma_{\mathrm{J}}^{n}}=\text { constant }, n=1, \ldots, N\right\} .
$$

Consider the following closed linear manifold of $\widetilde{\mathcal{X}}$,

$$
\widetilde{\mathcal{W}}(I):=\left\{\mathbf{G} \in \widetilde{\mathcal{X}}: \int_{\Gamma_{\mathbf{J}}} \operatorname{curl} \mathbf{G} \cdot \mathbf{n} \bar{\nu}=\int_{\Gamma_{\mathbf{J}}} I \bar{\nu} \quad \forall \nu \in \widetilde{\mathcal{L}}\right\},
$$

and its corresponding associated subspace

$$
\widetilde{\mathcal{W}}(0)=\left\{\mathbf{G} \in \widetilde{\mathcal{X}}: \int_{\Gamma_{\mathbf{J}}} \operatorname{curl} \mathbf{G} \cdot \mathbf{n} \bar{\nu}=0 \quad \forall \nu \in \widetilde{\mathcal{L}}\right\} .
$$

Notice that $\mathbf{G} \in \widetilde{\mathcal{W}}(\mathbf{I})$ if and only if

$$
\mathbf{G} \in \widetilde{\mathcal{X}}, \quad \operatorname{curl} \mathbf{G} \cdot \mathbf{n}=0 \text { on } \Gamma_{\mathbf{J}}^{0}, \quad \text { and } \int_{\Gamma_{\mathbf{J}}^{n}} \operatorname{curl} \mathbf{G} \cdot \mathbf{n}=I_{n}, n=1, \ldots, N .
$$

We introduce the following problem:

Problem 4.6. Given $\mathbf{I} \in \mathbb{C}^{N}$, find $\mathbf{H}_{\mathbf{I}} \in \widetilde{\mathcal{W}}(I)$ such that

$$
a\left(\mathbf{H}_{\mathbf{I}}, \mathbf{G}\right)=0 \quad \forall \mathbf{G} \in \widetilde{\mathcal{W}}(0) .
$$

In a way similar to how we did in the previous sections, we consider a mixed problem for handling the constraints of $\widetilde{\mathcal{W}}(I)$ and $\widetilde{\mathcal{W}}(0)$ : 
Problem 4.7. Given $\mathbf{I} \in \mathbb{C}^{N}$, find $\mathbf{H}_{\mathbf{I}} \in \widetilde{\mathcal{X}}$ and $\lambda_{\mathbf{I}} \in \widetilde{\mathcal{L}}$ such that

$$
\begin{aligned}
& a\left(\mathbf{H}_{\mathbf{I}}, \mathbf{G}\right)+b\left(\overline{\mathbf{G}}, \lambda_{\mathbf{I}}\right)=0 \quad \forall \mathbf{G} \in \widetilde{\mathcal{X}}, \\
& b\left(\mathbf{H}_{\mathbf{I}}, \nu\right)=\int_{\Gamma_{\mathbf{J}}} I \bar{\nu} \quad \forall \nu \in \widetilde{\mathcal{L}}
\end{aligned}
$$

Although $a$ is not $\widetilde{\mathcal{X}}$-elliptic in $\widetilde{\mathcal{W}}(0)$, we are able to prove the following result:

Theorem 4.8. Given $\mathbf{I} \in \mathbb{C}^{N}$, let us assume that the solution $\left(\mathbf{H}_{\mathbf{I}}, \lambda_{\mathbf{I}}\right)$ of Problem 4.3 satisfies $\operatorname{curl} \mathbf{H}_{\mathbf{I}} \cdot \mathbf{n} \in \mathrm{L}^{2}\left(\Gamma_{\mathbf{J}}\right)$. Then $\mathbf{H}_{\mathbf{I}}$ is the unique solution of Problem 4.6 and $\left(\mathbf{H}_{\mathbf{I}}, \lambda_{\mathbf{I}}\right)$ the only solution of Problem 4.7.

Proof. First $\mathbf{H}_{\mathbf{I}} \in \widetilde{\mathcal{X}}$, because $\operatorname{curl} \mathbf{H}_{\mathbf{I}} \cdot \mathbf{n} \in \mathrm{L}^{2}\left(\Gamma_{\mathbf{J}}\right)$. Since $\left(\mathbf{H}_{\mathbf{I}}, \lambda_{\mathbf{I}}\right)$ satisfies (4.1), $\lambda_{\mathbf{I}} \in \mathcal{L} \subset \widetilde{\mathcal{L}}$, and $\widetilde{\mathcal{X}} \subset \mathcal{X}$, we have that $\left(\mathbf{H}_{\mathbf{I}}, \lambda_{\mathbf{I}}\right)$ satisfies (4.4) too. Moreover, as shown in Remark 4.5 (4.2) implies that $\operatorname{curl}_{\mathbf{1}} \mathbf{H}_{\mathbf{I}} \cdot \mathbf{n}=0$ and $\int_{\Gamma_{\mathbf{J}}^{n}} \operatorname{curl}_{\mathbf{I}} \mathbf{H}_{\mathbf{I}} \cdot \mathbf{n}=I_{n}$ for $n=1, \ldots, N$. Therefore, (4.5) holds true. Thus, we have proved that $\left(\mathbf{H}_{\mathbf{I}}, \lambda_{\mathbf{I}}\right)$ is solution of Problem 4.7 .

On the other hand, for any solution $\left(\mathbf{H}_{\mathbf{I}}, \lambda_{\mathbf{I}}\right)$ of Problem 4.7, clearly $\mathbf{H}_{\mathbf{I}}$ is a solution of Problem 4.6. Moreover, this problem has at most one solution. Indeed, let $\mathbf{H}_{\mathbf{I}}$ and $\mathbf{H}_{\mathbf{I}}^{\prime}$ be solutions of Problem 4.6. Then $\mathbf{H}_{\mathbf{I}}-\mathbf{H}_{\mathbf{I}}^{\prime} \in \widetilde{\mathcal{W}}(0)$ and, hence, $a\left(\mathbf{H}_{\mathbf{I}}-\mathbf{H}_{\mathbf{I}}^{\prime}, \mathbf{H}_{\mathbf{I}}-\mathbf{H}_{\mathbf{I}}^{\prime}\right)=0$. Therefore, since $a$ is $\mathcal{X}$-elliptic, $\mathbf{H}_{\mathbf{I}}=\mathbf{H}_{\mathbf{I}}^{\prime}$.

Thus, any two solutions of Problem 4.7 must have a coinciding magnetic field $\mathbf{H}_{\mathbf{I}}$. Hence, to end the proof, we only need to show that the corresponding Lagrange multiplier $\lambda_{\mathbf{I}}$ is also unique. In fact, the latter is an immediate consequence of the inf-sup condition that $b$ satisfies in $\widetilde{\mathcal{M}}$ (and a fortiori in $\widetilde{\mathcal{L}} \subset \widetilde{\mathcal{M}}$ ), which has been shown in the proof of Theorem 3.10

Remark 4.9. As shown in the proof of the theorem above, if the solution $\left(\mathbf{H}_{\mathbf{I}}, \lambda_{\mathbf{I}}\right)$ of Problem 4.3 satisfies $\operatorname{curl}_{\mathbf{l}} \mathbf{H}_{\mathbf{I}} \cdot \mathbf{n} \in \mathrm{L}^{2}\left(\Gamma_{\mathbf{J}}\right)$, then it satisfies the boundary condition (2.5):

$$
\int_{\Gamma_{\mathbf{J}}^{n}} \operatorname{curl}_{\mathbf{I}} \cdot \mathbf{n}=I_{n} \quad \text { on } \Gamma_{\mathrm{J}}^{n}, \quad n=1, \ldots, N
$$

Thus, according to Remark 4.5, $\mathbf{H}_{\mathbf{I}}$ satisfies all the boundary conditions (2.4)-(2.8)

\section{Finite element discretization}

In this section we introduce discretizations of the different mixed problems introduced above and study their convergence properties. To this goal, we assume that $\Omega$ is a Lipschitz polyhedron and that $\Gamma_{\mathrm{J}}^{n}$ are polyhedral surfaces for all $n=0, \ldots, N$. Consequently, $\Gamma_{\mathrm{E}}$ is also a polyhedral surface. We also assume that $\sigma$ is piecewise smooth (e.g., $\mathcal{C}^{2}$ ) on a polyhedral partition of $\Omega$.

We consider a family of shape-regular tetrahedral meshes $\left\{\mathcal{T}_{h}\right\}$ of $\Omega$ where, as usual, $h$ denotes the corresponding mesh size. We assume that the meshes are compatible with the splittings of the domain boundary in the sense that, $\forall K \in \mathcal{T}_{h}$ with a face $T$ lying on $\partial \Omega$,

- either $T \subset \bar{\Gamma}_{\mathbf{E}}$ or $T \subset \bar{\Gamma}_{\mathrm{J}}^{n}$ for some $n=0, \ldots, N$, and

$-\left.\sigma\right|_{T}$ is smooth.

The magnetic field, which is a function of $\mathrm{H}(\mathbf{c u r l}, \Omega)$ in all of the problems, is discretized with lowest-order Nédélec edge finite elements (see [19]). We recall their 
definition. For each tetrahedron $K \in \mathcal{T}_{h}$, let

$$
\mathcal{N}(K):=\left\{\mathbf{G}_{h} \in \mathcal{P}_{1}(K)^{3}: \mathbf{G}_{h}(\mathbf{x})=\mathbf{a} \times \mathbf{x}+\mathbf{b}, \mathbf{a}, \mathbf{b} \in \mathbb{C}^{3}, \mathbf{x} \in K\right\} .
$$

Then, fields in $\mathrm{H}(\mathbf{c u r l}, \Omega)$ are approximated in the finite-dimensional space

$$
\mathcal{N}_{h}(\Omega):=\left\{\mathbf{G}_{h} \in \mathrm{H}(\operatorname{curl}, \Omega):\left.\mathbf{G}_{h}\right|_{K} \in \mathcal{N}(K) \forall K \in \mathcal{T}_{h}\right\} .
$$

Since $\mathcal{N}_{h}(\Omega) \subset \widetilde{\mathcal{X}}$ too, the main difference between the discretizations of Problems [3.3, 3.9, 4.3] and 4.7] is the space used to discretize the Lagrange multiplier.

Let $\mathcal{T}_{h}{ }^{\Gamma_{\mathbf{J}}}$ be the triangular mesh induced by $\mathcal{T}_{h}$ on the polyhedral surface $\Gamma_{\mathbf{J}}$ and consider the following finite-dimensional spaces:

$$
\begin{aligned}
& \mathcal{Q}_{h}^{1}\left(\Gamma_{\mathbf{J}}\right):=\left\{q_{h} \in \mathrm{H}_{0}^{1}\left(\Gamma_{\mathbf{J}}\right):\left.q_{h}\right|_{T} \in \mathcal{P}_{1}(T) \forall T \in \mathcal{T}_{h}^{\Gamma_{\mathbf{J}}}\right\}, \\
& \mathcal{Q}_{h}^{0}\left(\Gamma_{\mathbf{J}}\right):=\left\{q_{h} \in \mathrm{L}^{2}\left(\Gamma_{\mathbf{J}}\right):\left.q_{h}\right|_{T} \in \mathcal{P}_{0}(T) \forall T \in \mathcal{T}_{h}^{\Gamma_{\mathbf{J}}}\right\} .
\end{aligned}
$$

These are, respectively, the spaces of piecewise linear continuous functions (vanishing on the boundary) and piecewise constant functions defined on $\mathcal{T}_{h}^{\Gamma_{\mathbf{J}}}$.

Let $\Pi_{h}: \mathrm{L}^{2}\left(\Gamma_{\mathrm{J}}\right) \longrightarrow \mathcal{Q}_{h}^{0}\left(\Gamma_{\mathrm{J}}\right)$ be the $\mathrm{L}^{2}\left(\Gamma_{\mathrm{J}}\right)$-orthogonal projection; that is,

$$
\forall f \in \mathrm{L}^{2}\left(\Gamma_{\mathbf{J}}\right),\left.\quad \Pi_{h} f\right|_{T}=\frac{1}{\operatorname{meas}(T)} \int_{T} f \quad \forall T \in \mathcal{T}_{h}^{\Gamma_{\mathbf{J}}} .
$$

If $\mathbf{G}$ is smooth enough for the boundary integrals below to make sense, its Nédélec interpolant $\mathbf{G}^{\mathrm{N}}$ is defined by

$$
\mathbf{G}^{\mathrm{N}} \in \mathcal{N}_{h}(\Omega): \quad \int_{\ell} \mathbf{G}^{\mathrm{N}} \cdot \mathbf{t}_{\ell}=\int_{\ell} \mathbf{G} \cdot \mathbf{t}_{\ell} \quad \forall \ell \text { edge of } \mathcal{T}_{h},
$$

where $\mathbf{t}_{\ell}$ denotes a unit vector tangent to the edge $\ell$. We recall in the following lemma some properties of this interpolant which has been essentially proved in [4] and $[3]$.

Lemma 5.1. The Nédélec interpolation operator

$$
\begin{aligned}
\mathcal{C}^{\infty}(\bar{\Omega})^{3} & \longrightarrow \mathcal{N}_{h}(\Omega) \\
\mathbf{G} & \longmapsto \mathbf{G}^{\mathrm{N}}
\end{aligned}
$$

extends uniquely to the space $\left\{\mathbf{G} \in \mathrm{H}^{r}(\Omega)^{3}: \operatorname{curl} \mathbf{G} \in \mathrm{H}^{s}(\Omega)^{3}\right\}$, for any $r>1 / 2$ and $s>0$, and the following error estimate holds true:

$$
\left\|\mathbf{G}-\mathbf{G}^{\mathrm{N}}\right\|_{\mathrm{H}(\mathbf{c u r l}, \Omega)} \leq C h^{\min \{1, r, s\}}\left[\|\mathbf{G}\|_{\mathrm{H}^{r}(\Omega)^{3}}+\|\mathbf{c u r l} \mathbf{G}\|_{\mathrm{H}^{s}(\Omega)^{3}}\right] .
$$

Furthermore, if curl $\mathbf{G} \cdot \mathbf{n} \in \mathrm{L}^{2}\left(\Gamma_{\mathbf{J}}\right)$, then

$$
\operatorname{curl~G} \mathbf{N}^{\mathrm{N}} \cdot \mathbf{n}=\Pi_{h}(\operatorname{curl} \mathbf{G} \cdot \mathbf{n}) \text { on } \Gamma_{\mathbf{J}} .
$$

Proof. Let $\mathbf{G} \in \mathrm{H}^{r}(\Omega)^{3}, r>1 / 2$, with curl $\mathbf{G} \in \mathrm{H}^{s}(\Omega)^{3}, s>0$. According to the Sobolev imbedding theorem and a trace theorem, for each $K \in \mathcal{T}_{h},\left.\mathbf{G}\right|_{K} \in \mathrm{L}^{p}(K)^{3}$, $\left.\operatorname{curl} \mathbf{G}\right|_{K} \in \mathrm{L}^{p}(K)^{3}$, and $\mathbf{G} \times\left.\mathbf{n}_{K}\right|_{\partial K} \in \mathrm{L}^{p}(\partial K)^{3}$, with $p>2$ depending on $r$ and $s$, and $\mathbf{n}_{K}$ being an outer unit normal to $\partial K$. Then, the extension of the Nédélec interpolation operator follows by applying Lemma 4.7 of 4 .

On the other hand, the arguments in the proof of Proposition 5.6 of [3] combined with Lemma 5.5 of the same reference yield the error estimate (5.1).

Finally, a density argument, the definition of $\mathbf{G}^{\mathrm{N}}$, and Stokes' Theorem yield

$$
\int_{T} \operatorname{curl} \mathbf{G}^{\mathrm{N}} \cdot \mathbf{n}=\int_{\partial T} \mathbf{G}^{\mathrm{N}} \cdot \mathbf{t}_{T}=\int_{\partial T} \mathbf{G} \cdot \mathbf{t}_{T}=\int_{T} \operatorname{curl} \mathbf{G} \cdot \mathbf{n} \quad \forall T \in \mathcal{T}_{h}^{\Gamma_{\mathbf{J}}}
$$


$\left(\mathbf{t}_{T}\right.$ being a unit vector tangent to $\left.\partial T\right)$. Hence, since $\mathbf{c u r l ~ G}^{\mathrm{N}} \cdot \mathbf{n} \in \mathcal{Q}_{h}^{0}\left(\Gamma_{\mathbf{J}}\right)$, we conclude the proof.

The following lemmas are used below to establish adequate inf-sup conditions.

Lemma 5.2. The application $\Pi_{h}: \mathcal{Q}_{h}^{1}\left(\Gamma_{\mathrm{J}}\right) \longrightarrow \mathcal{Q}_{h}^{0}\left(\Gamma_{\mathrm{J}}\right)$ is one-to-one.

Proof. To prove the result, we take $\nu_{h} \in \mathcal{Q}_{h}^{1}\left(\Gamma_{\mathbf{J}}\right)$ such that $\int_{T} \nu_{h}=0 \forall T \in \mathcal{T}_{h}^{\Gamma_{\mathbf{J}}}$ and prove that $\nu_{h}=0$ on $\Gamma_{J}$. We do this by induction on the number $m$ of triangles of the mesh.

If $m=1$, clearly $\nu_{h}=0$. Assuming the result true for any mesh of $(m-1)$ triangles, we are going to show that it is also true for a mesh $\mathcal{T}_{h}^{\Gamma_{\mathbf{J}}}$ of $m$ triangles.

Our geometric assumptions imply that $\partial \Gamma_{J}$ is a polygonal curve of positive onedimensional measure. Then, in any triangular mesh there is always a triangle with at least one edge on the boundary $\partial \Gamma_{\mathrm{J}}$. Let $T \in \mathcal{T}_{h}^{\Gamma_{\mathbf{J}}}$ be one such triangle. We have $\nu_{h}=0$ on $T$, since $\int_{T} \nu_{h}=0$.

Let $\Gamma_{\mathbf{J}}^{\prime}:=\Gamma_{\mathbf{J}} \backslash T$. Then $\mathcal{T}_{h}^{\Gamma_{\mathbf{J}}^{\prime}}:=\mathcal{T}_{h}^{\Gamma_{\mathbf{J}}} \backslash\{T\}$ is a mesh on $\Gamma_{\mathbf{J}}^{\prime}$ of $(m-1)$ triangles. Since $\nu_{h}=0$ on $T$, we have that $\left.\nu_{h}\right|_{\Gamma_{\mathbf{J}}^{\prime}} \in \mathcal{Q}_{h}^{1}\left(\Gamma_{\mathrm{J}}^{\prime}\right)$. Hence, since $\int_{T} \nu_{h}=0 \forall T \in \mathcal{T}_{h}^{\Gamma_{\mathrm{J}}^{\prime}}$, we apply the inductive assumption and conclude that $\nu_{h}=0$ on $\Gamma_{\mathbf{J}}^{\prime}$. Therefore $\nu_{h}=0$ on $\Gamma_{\mathrm{J}}$ and we conclude the proof.

Lemma 5.3. Given $q_{h} \in \mathcal{Q}_{h}^{0}\left(\Gamma_{\mathbf{J}}\right), \exists \mathbf{G}_{h} \in \mathcal{N}_{h}(\Omega)$ such that $\mathbf{c u r l} \mathbf{G}_{h} \cdot \mathbf{n}=q_{h}$ on $\Gamma_{\mathbf{J}}$. Furthermore, $\left\|\mathbf{G}_{h}\right\|_{\mathrm{H}(\mathbf{c u r l}, \Omega)^{3}} \leq C\left\|q_{h}\right\|_{\mathrm{L}^{2}\left(\Gamma_{\mathbf{J}}\right)}$.

Proof. Let $\mathbf{G} \in \mathcal{X}$ be the vector field associated with $q_{h}$ by Lemma 2.1. Since $q_{h} \in \mathrm{L}^{2}\left(\Gamma_{\mathbf{J}}\right)$, we have that $\mathbf{G} \in \mathrm{H}^{r}(\Omega)^{3}$ with $r>1 / 2$, curl $\mathbf{G} \in \mathrm{H}^{s}(\Omega)^{3}$ with $s>0$, and (2.16)-(2.17) hold true. Hence, because of Lemma 5.1, the Nédélec interpolant $\mathbf{G}^{\mathrm{N}} \in \mathcal{N}_{h}(\Omega)$ is well defined.

We take $\mathbf{G}_{h}=\mathbf{G}^{\mathrm{N}}$. Then, because of Lemma 5.1, we have that $\operatorname{curl} \mathbf{G}_{h}$. $\mathbf{n}=\Pi_{h}(\operatorname{curl} \mathbf{G} \cdot \mathbf{n})=\Pi_{h} q_{h}=q_{h}$ on $\Gamma_{\boldsymbol{J}}$. Finally, the estimate to be proved is a consequence of (5.1), (2.16), and (2.17).

In the following subsections we introduce discretizations for each of the different mixed problems, 3.3 3.9, 4.3 and 4.7 and study their convergence properties. $\left(H_{n}, \lambda_{n}\right)$ is used to denote the solutions of all these discrete problems, although in general they are not the same. We do this to simplify the notation. No confusion should arise because we consider only one discrete problem in each subsection.

5.1. Discretizing Problem 3.3. In this case, since the Lagrange multiplier $\lambda \in$ $\mathrm{H}_{00}^{1 / 2}\left(\Gamma_{\mathrm{J}}\right)$, we use piecewise linear continuous finite elements for its discretization. Let

$$
\mathcal{X}_{h}:=\mathcal{N}_{h}(\Omega) \quad \text { and } \quad \mathcal{M}_{h}:=\mathcal{Q}_{h}^{1}\left(\Gamma_{\mathbf{J}}\right) \text {. }
$$

In what follows, we prove that $b$ satisfies an inf-sup condition on these discrete spaces, although with a constant which may depend on the mesh size $h$.

Lemma 5.4. There exists $\beta_{h}>0$ such that

$$
\sup _{\mathbf{G}_{h} \in \mathcal{X}_{h}} \frac{\left|b\left(\mathbf{G}_{h}, \nu_{h}\right)\right|}{\left\|\mathbf{G}_{h}\right\|_{\mathcal{X}}} \geq \beta_{h}\left\|\nu_{h}\right\|_{\mathcal{M}} \quad \forall \nu_{h} \in \mathcal{M}_{h}
$$


Proof. Since $\mathcal{X}_{h}$ and $\mathcal{M}_{h}$ are finite-dimensional, we only have to prove that, given a non-vanishing $\nu_{h} \in \mathcal{M}_{h}, \exists \widetilde{\mathbf{G}}_{h} \in \mathcal{X}_{h}$ such that $\int_{\Gamma_{\mathbf{J}}}$ curl $\widetilde{\mathbf{G}}_{h} \cdot \mathbf{n} \bar{\nu}_{h} \neq 0$.

To prove this, notice that $\Pi_{h} \nu_{h} \in \mathcal{Q}_{h}^{0}\left(\Gamma_{\mathrm{J}}\right)$ does not vanish either, because of Lemma5.2. Now, according to Lemma 5.3, $\exists \widetilde{\mathbf{G}}_{h} \in \mathcal{X}_{h}$ satisfying curl $\widetilde{\mathbf{G}}_{h} \cdot \mathbf{n}=\Pi_{h} \nu_{h}$ on $\Gamma_{\mathrm{J}}$. Then, we have

$$
\int_{\Gamma_{\mathbf{J}}} \operatorname{curl} \widetilde{\mathbf{G}}_{h} \cdot \mathbf{n} \bar{\nu}_{h}=\int_{\Gamma_{\mathbf{J}}} \Pi_{h} \nu_{h} \bar{\nu}_{h}=\int_{\Gamma_{\mathbf{J}}}\left|\Pi_{h} \nu_{h}\right|^{2}>0
$$

and we conclude the proof.

Remark 5.5. We have not been able to prove that the constant $\beta_{h}$ remains bounded away from zero when $h$ goes to 0 . Actually, our numerical experiments seem to show that $\beta_{h}$ converges to zero with a linear dependence on $h$.

If we approximate Problem 3.3 with the finite element spaces $\mathcal{X}_{h}$ and $\mathcal{M}_{h}$, Lemma 5.4 allows us to show that the resulting discrete problem has a unique solution. However, since the inf-sup condition is not uniform in $h$, the approximating properties of such a scheme depend on $\inf _{\mathbf{G}_{h} \in \mathcal{V}_{h}(g)}\left\|\mathbf{H}_{g}-\mathbf{G}_{h}\right\|_{\mathcal{X}}$, with $\mathcal{V}_{h}(g):=\left\{\mathbf{G}_{h} \in \mathcal{X}_{h}: b\left(\mathbf{G}_{h}, \nu_{h}\right)=\left\langle g, \nu_{h}\right\rangle_{\Gamma_{\mathbf{J}}} \forall \nu_{h} \in \mathcal{M}_{h}\right\}$ (see Theorem II.1.1 of [14]), and it is not clear that this infimum can be estimated for smooth $\mathbf{H}_{g}$ either.

To avoid this drawback, we introduce a variational crime in the discrete formulation which allows us to prove error estimates under an appropriate mild smoothness assumption on $\mathbf{H}_{g}$. This variational crime can be seen as a kind of quadrature rule for $\left\langle g, \nu_{h}\right\rangle_{\Gamma_{\mathbf{J}}}$. It consists of substituting $g$ with $\Pi_{h} g$ in this term. This is applicable whenever the data $g$ is an $\mathrm{L}^{2}\left(\Gamma_{\mathbf{J}}\right)$-function. In such a case, we define the following discrete problem:

Problem 5.6. Given $g \in \mathrm{L}^{2}\left(\Gamma_{\mathbf{J}}\right)$, find $\mathbf{H}_{h} \in \mathcal{X}_{h}$ and $\lambda_{h} \in \mathcal{M}_{h}$ such that

$$
\begin{aligned}
& a\left(\mathbf{H}_{h}, \mathbf{G}_{h}\right)+b\left(\overline{\mathbf{G}}_{h}, \lambda_{h}\right)=0 \quad \forall \mathbf{G}_{h} \in \mathcal{X}_{h}, \\
& b\left(\mathbf{H}_{h}, \nu_{h}\right)=\int_{\Gamma_{\mathbf{J}}} \Pi_{h} g \bar{\nu}_{h} \quad \forall \nu_{h} \in \mathcal{M}_{h} .
\end{aligned}
$$

The following theorem yields an error estimate for the approximate magnetic field $\mathbf{H}_{h}$. Its proof follows similar arguments to those used to prove Theorem II.1.1 of [14].

Theorem 5.7. Given $g \in \mathrm{L}^{2}\left(\Gamma_{\mathrm{J}}\right)$, let us assume that the solution $\left(\mathbf{H}_{g}, \lambda_{g}\right)$ of Problem 3.3 satisfies $\mathbf{H}_{g} \in \mathrm{H}^{r}(\mathbf{c u r l}, \Omega)$ with $1 / 2<r \leq 1$. Then, Problem 5.6 has a unique solution $\left(\mathbf{H}_{h}, \lambda_{h}\right)$ and the following error estimate holds true:

$$
\left\|\mathbf{H}_{g}-\mathbf{H}_{h}\right\|_{\mathcal{X}} \leq C h^{r}\left\|\mathbf{H}_{g}\right\|_{\mathrm{H}^{r}(\mathbf{c u r l}, \Omega)} .
$$

Proof. The discrete Problem 5.6 has a unique solution, because $a$ is elliptic in $\mathcal{X}_{h}$ and $b$ satisfies the inf-sup condition of Lemma 5.4

According to Lemma [5.1] the Nédélec interpolant $\mathbf{H}_{g}^{\mathrm{N}} \in \mathcal{X}_{h}$ is well defined and

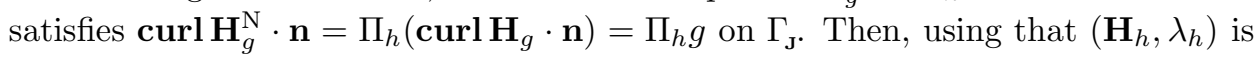
the solution of Problem 5.6. we have

$$
b\left(\mathbf{H}_{h}-\mathbf{H}_{g}^{\mathrm{N}}, \nu_{h}\right)=0 \quad \forall \nu_{h} \in \mathcal{M}_{h}
$$

and

$a\left(\mathbf{H}_{h}-\mathbf{H}_{g}^{\mathrm{N}}, \mathbf{H}_{h}-\mathbf{H}_{g}^{\mathrm{N}}\right)=-b\left(\overline{\mathbf{H}}_{h}-\overline{\mathbf{H}}_{g}^{\mathrm{N}}, \lambda_{h}\right)-a\left(\mathbf{H}_{g}^{\mathrm{N}}, \mathbf{H}_{h}-\mathbf{H}_{g}^{\mathrm{N}}\right)=-a\left(\mathbf{H}_{g}^{\mathrm{N}}, \mathbf{H}_{h}-\mathbf{H}_{g}^{\mathrm{N}}\right)$. 
On the other hand, since $\mathbf{H}_{h}-\mathbf{H}_{g}^{\mathrm{N}} \in \mathcal{X}_{h} \subset \mathcal{X}$, we can take $\mathbf{G}=\mathbf{H}_{h}-\mathbf{H}_{g}^{\mathrm{N}}$ in Problem 3.3 and obtain

$$
a\left(\mathbf{H}_{g}, \mathbf{H}_{h}-\mathbf{H}_{g}^{\mathrm{N}}\right)+b\left(\overline{\mathbf{H}}_{h}-\overline{\mathbf{H}}_{g}^{\mathrm{N}}, \lambda_{g}\right)=0 .
$$

Combining this with the equations above, we have

$$
\begin{aligned}
& a\left(\mathbf{H}_{h}-\mathbf{H}_{g}^{\mathrm{N}}, \mathbf{H}_{h}-\mathbf{H}_{g}^{\mathrm{N}}\right)= a\left(\mathbf{H}_{g}-\mathbf{H}_{g}^{\mathrm{N}}, \mathbf{H}_{h}-\mathbf{H}_{g}^{\mathrm{N}}\right)+b\left(\overline{\mathbf{H}}_{h}-\overline{\mathbf{H}}_{g}^{\mathrm{N}}, \lambda_{g}\right) \\
&=a\left(\mathbf{H}_{g}-\mathbf{H}_{g}^{\mathrm{N}}, \mathbf{H}_{h}-\mathbf{H}_{g}^{\mathrm{N}}\right)+b\left(\overline{\mathbf{H}}_{h}-\overline{\mathbf{H}}_{g}^{\mathrm{N}}, \lambda_{g}-\nu_{h}\right) \\
& \forall \nu_{h} \in \mathcal{M}_{h} .
\end{aligned}
$$

Hence, the $\mathcal{X}$-ellipticity of $a$ (see (3.2)) together with the continuity of $a$ and $b$ yield

$$
\left\|\mathbf{H}_{h}-\mathbf{H}_{g}^{\mathrm{N}}\right\|_{\mathcal{X}} \leq \frac{1}{\alpha}\left(\|a\|_{\mathcal{X} \times \mathcal{X}}\left\|\mathbf{H}_{g}-\mathbf{H}_{g}^{\mathrm{N}}\right\|_{\mathcal{X}}+\|b\|_{\mathcal{X} \times \mathcal{M}}\left\|\lambda_{g}-\nu_{h}\right\|_{\mathcal{M}}\right)
$$

which combined with the triangular inequality lead to

$$
\begin{array}{r}
\left\|\mathbf{H}_{g}-\mathbf{H}_{h}\right\|_{\mathcal{X}} \leq\left(1+\frac{1}{\alpha}\|a\|_{\mathcal{X} \times \mathcal{X}}\right)\left\|\mathbf{H}_{g}-\mathbf{H}_{g}^{\mathrm{N}}\right\|_{\mathcal{X}}+\frac{1}{\alpha}\|b\|_{\mathcal{X} \times \mathcal{M}}\left\|\lambda_{g}-\nu_{h}\right\|_{\mathcal{M}} \\
\forall \nu_{h} \in \mathcal{M}_{h}
\end{array}
$$

Now, by virtue of Corollary 3.6 the Lagrange interpolant of $\lambda_{g}, \lambda_{g}^{\mathrm{L}} \in \mathcal{M}_{h}$, is well defined and satisfies

$$
\left\|\lambda_{g}-\lambda_{g}^{\mathrm{L}}\right\|_{\mathcal{M}} \leq C h^{r}\left\|\mathbf{H}_{g}\right\|_{\mathrm{H}^{r}(\operatorname{curl}, \Omega)},
$$

because of standard interpolation results and (3.11).

Finally, the two above inequalities and (5.1) yield the error estimate.

5.2. Discretizing Problem 3.9. In this case we use the finite-dimensional spaces

$$
\widetilde{\mathcal{X}}_{h}:=\mathcal{N}_{h}(\Omega) \quad \text { and } \quad \widetilde{\mathcal{M}}_{h}:=\mathcal{Q}_{h}^{0}\left(\Gamma_{\mathrm{J}}\right)
$$

to define the following discrete problem:

Problem 5.8. Given $g \in \mathrm{L}^{2}\left(\Gamma_{\mathbf{J}}\right)$, find $\mathbf{H}_{h} \in \widetilde{\mathcal{X}}_{h}$ and $\lambda_{h} \in \widetilde{\mathcal{M}}_{h}$ such that

$$
\begin{aligned}
& a\left(\mathbf{H}_{h}, \mathbf{G}_{h}\right)+b\left(\overline{\mathbf{G}}_{h}, \lambda_{h}\right)=0 \quad \forall \mathbf{G}_{h} \in \widetilde{\mathcal{X}}_{h}, \\
& b\left(\mathbf{H}_{h}, \nu_{h}\right)=\int_{\Gamma_{\mathbf{J}}} g \bar{\nu}_{h} \quad \forall \nu_{h} \in \widetilde{\mathcal{M}}_{h} .
\end{aligned}
$$

The following lemma shows that $b$ satisfies a uniform inf-sup condition on these discrete spaces.

Lemma 5.9. There exists $\widetilde{\beta}_{*}>0$ such that

$$
\sup _{\mathbf{G}_{h} \in \widetilde{\mathcal{X}}_{h}} \frac{\left|b\left(\mathbf{G}_{h}, \nu_{h}\right)\right|}{\left\|\mathbf{G}_{h}\right\|_{\widetilde{\mathcal{X}}}} \geq \widetilde{\beta}_{*}\left\|\nu_{h}\right\|_{\widetilde{\mathcal{M}}} \quad \forall \nu_{h} \in \widetilde{\mathcal{M}}_{h} .
$$

Proof. Given $\nu_{h} \in \widetilde{\mathcal{M}}_{h}$, let $\widetilde{\mathbf{G}}_{h} \in \mathcal{N}_{h}(\Omega)$ be the vector field associated with $\nu_{h}$ by Lemma 5.3. Then, since $\operatorname{curl} \widetilde{\mathbf{G}}_{h} \cdot \mathbf{n}=\nu_{h}$ on $\Gamma_{\mathbf{J}}$, we have

$$
\left\|\widetilde{\mathbf{G}}_{h}\right\|_{\widetilde{\mathcal{X}}}=\left[\left\|\widetilde{\mathbf{G}}_{h}\right\|_{\mathrm{H}(\operatorname{curl}, \Omega)}^{2}+\left\|\operatorname{curl} \widetilde{\mathbf{G}}_{h} \cdot \mathbf{n}\right\|_{\mathrm{L}^{2}\left(\Gamma_{\mathbf{J}}\right)}^{2}\right]^{1 / 2} \leq C\left\|\nu_{h}\right\|_{\widetilde{\mathcal{M}}}
$$


Hence,

$$
\sup _{\mathbf{G}_{h} \in \widetilde{\mathcal{X}}_{h}} \frac{\left|b\left(\mathbf{G}_{h}, \nu_{h}\right)\right|}{\left\|\mathbf{G}_{h}\right\|_{\widetilde{\mathcal{X}}}} \geq \frac{\left|\int_{\Gamma_{\mathbf{J}}} \operatorname{curl} \widetilde{\mathbf{G}}_{h} \cdot \mathbf{n} \bar{\nu}_{h}\right|}{\left\|\widetilde{\mathbf{G}}_{h}\right\|_{\widetilde{\mathcal{X}}}} \geq \frac{\int_{\Gamma_{\mathbf{J}}}\left|\nu_{h}\right|^{2}}{C\left\|\nu_{h}\right\|_{\widetilde{\mathcal{M}}}}=\frac{1}{C}\left\|\nu_{h}\right\|_{\widetilde{\mathcal{M}}},
$$

which yields the lemma with $\widetilde{\beta}_{*}=\frac{1}{C}$.

The following theorem shows existence and uniqueness of solution for this problem and establishes error estimates for the magnetic field and the Lagrange multiplier:

Theorem 5.10. Given $g \in \mathrm{L}^{2}\left(\Gamma_{\mathrm{J}}\right)$, Problem 5.8 has a unique solution $\left(\mathbf{H}_{h}, \lambda_{h}\right)$ and, if $\left(\mathbf{H}_{g}, \lambda_{g}\right)$ is the solution of Problem [3.9, the following estimate holds true:

$$
\left\|\mathbf{H}_{g}-\mathbf{H}_{h}\right\|_{\widetilde{\mathcal{X}}}+\left\|\lambda_{g}-\lambda_{h}\right\|_{\widetilde{\mathcal{M}}} \leq C\left(\inf _{\mathbf{G}_{h} \in \widetilde{\mathcal{X}}_{h}}\left\|\mathbf{H}_{g}-\mathbf{G}_{h}\right\|_{\widetilde{\mathcal{X}}}+\inf _{\nu_{h} \in \widetilde{\mathcal{M}}_{h}}\left\|\lambda_{g}-\nu_{h}\right\|_{\widetilde{\mathcal{M}}}\right) .
$$

Furthermore, if $\mathbf{H}_{g} \in \mathrm{H}^{r}(\mathbf{c u r l}, \Omega)$ with $1 / 2<r \leq 1$, then

$$
\left\|\mathbf{H}_{g}-\mathbf{H}_{h}\right\|_{\mathcal{X}} \leq C h^{r}\left\|\mathbf{H}_{g}\right\|_{\mathrm{H}^{r}(\operatorname{curl}, \Omega)} .
$$

Proof. Let

$$
\widetilde{\mathcal{V}}_{h}(0):=\left\{\mathbf{G}_{h} \in \widetilde{\mathcal{X}}_{h}: \int_{\Gamma_{\mathbf{J}}} \operatorname{curl} \mathbf{G}_{h} \cdot \mathbf{n} \bar{\nu}_{h}=0 \quad \forall \nu_{h} \in \widetilde{\mathcal{M}}_{h}\right\} .
$$

Notice that $\widetilde{\mathcal{V}}_{h}(0) \subset \widetilde{\mathcal{V}}(0)$; indeed, since $\operatorname{curl} \mathbf{G}_{h} \cdot \mathbf{n} \in \widetilde{\mathcal{M}}_{h}$ for $\mathbf{G}_{h} \in \widetilde{\mathcal{X}}_{h}$, we know that $\mathbf{G}_{h}$ belongs to $\widetilde{\mathcal{V}}_{h}(0)$ if and only if $\operatorname{curl} \mathbf{G}_{h} \cdot \mathbf{n}=0$ on $\Gamma_{\boldsymbol{J}}$. Consequently, $a$ is $\widetilde{\mathcal{X}}$ elliptic in $\widetilde{\mathcal{V}}_{h}(0)$ with the same ellipticity constant $\alpha$ of (3.2). Then, since $b$ satisfies the inf-sup condition of Lemma[5.9, a straightforward application of Theorem II.1.1 of [14] allows us to prove the estimate (5.2).

On the other hand, if $\mathbf{H}_{g} \in \mathrm{H}^{r}(\mathbf{c u r l}, \Omega)$ with $1 / 2<r \leq 1$, according to Lemma 5.1 the Nédélec interpolant $\mathbf{H}_{g}^{\mathrm{N}} \in \widetilde{\mathcal{X}}_{h}$ is well defined and satisfies $\mathbf{c u r l}_{\mathbf{H}} \mathbf{H}_{g}^{\mathrm{N}} \cdot \mathbf{n}=$ $\Pi_{h}\left(\operatorname{curl} \mathbf{H}_{g} \cdot \mathbf{n}\right)=\Pi_{h} g$ on $\Gamma_{\mathbf{J}}$. Then, since $\left(\mathbf{H}_{h}, \lambda_{h}\right)$ is the solution of Problem 5.8, we have $b\left(\mathbf{H}_{h}-\mathbf{H}_{g}^{\mathrm{N}}, \nu_{h}\right)=0 \forall \nu_{h} \in \mathcal{M}_{h}$. Hence, $\mathbf{H}_{h}-\mathbf{H}_{g}^{\mathrm{N}} \in \widetilde{\mathcal{V}}_{h}(0) \subset \widetilde{\mathcal{V}}(0)$. Consequently, by taking $\mathbf{H}_{h}-\mathbf{H}_{g}^{\mathrm{N}}$ as test function in Problems 3.9 and 5.8 we obtain

$$
a\left(\mathbf{H}_{h}-\mathbf{H}_{g}^{\mathrm{N}}, \mathbf{H}_{h}-\mathbf{H}_{g}^{\mathrm{N}}\right)=a\left(\mathbf{H}_{g}-\mathbf{H}_{g}^{\mathrm{N}}, \mathbf{H}_{h}-\mathbf{H}_{g}^{\mathrm{N}}\right) .
$$

Then, since $a$ is $\tilde{\mathcal{X}}$-elliptic in $\tilde{\mathcal{V}}_{h}(0)$ and continuous in $\mathcal{X} \times \boldsymbol{\mathcal { X }}$, we have

$$
\left\|\mathbf{H}_{h}-\mathbf{H}_{g}^{\mathrm{N}}\right\|_{\widetilde{\mathcal{X}}} \leq \frac{1}{\alpha}\|a\|_{\mathcal{X} \times \mathcal{X}}\left\|\mathbf{H}_{g}-\mathbf{H}_{g}^{\mathrm{N}}\right\|_{\mathcal{X}}
$$

Finally, from this estimate, the triangle inequality, and (5.1), we obtain (5.3).

Remark 5.11. Estimate (5.3) cannot be directly obtained from (5.2) without further assumptions on the smoothness of the input current density flux $g=\operatorname{curl} \mathbf{H}_{g} \cdot \mathbf{n}$ on $\Gamma_{\boldsymbol{J}}$. In fact, (5.2) depends on $\inf _{\mathbf{G}_{h} \in \widetilde{\mathcal{X}}_{h}}\left\|\mathbf{H}_{g}-\mathbf{G}_{h}\right\|_{\widetilde{\mathcal{X}}}$, which in its turn involves $\left\|\operatorname{curl}_{\mathbf{H}_{g}} \cdot \mathbf{n}-\operatorname{curl} \mathbf{G}_{h} \cdot \mathbf{n}\right\|_{\mathrm{L}^{2}\left(\Gamma_{\mathbf{J}}\right)}$. For $\mathbf{H}_{g} \in \mathrm{H}^{r}(\mathbf{c u r l}, \Omega)$ with $1 / 2<r \leq 1$, $\operatorname{curl} \mathbf{H}_{g} \cdot \mathbf{n} \in \mathrm{H}^{r-1 / 2}\left(\Gamma_{\mathbf{J}}\right)$ and, hence, for its Nédélec interpolant $\mathbf{H}_{g}^{\mathrm{N}} \in \widetilde{\mathcal{X}}_{h}$ we only have $\left\|\mathbf{H}_{g}-\mathbf{H}_{g}^{\mathrm{N}}\right\|_{\widetilde{\mathcal{X}}} \leq C h^{r-1 / 2}\left\|\mathbf{H}_{g}\right\|_{\mathrm{H}^{r}(\mathbf{c u r l}, \Omega)}$. 
5.3. Discretizing Problem 4.3. In this case we use the finite-dimensional spaces

$$
\mathcal{X}_{h}:=\mathcal{N}_{h}(\Omega) \quad \text { and } \quad \mathcal{L}_{h}:=\left\{\nu_{h} \in \mathcal{Q}_{h}^{1}\left(\Gamma_{\mathbf{J}}\right):\left.\nu_{h}\right|_{\Gamma_{\mathbf{J}}^{n}}=\text { constant, } n=1, \ldots, N\right\}
$$

to define the following discrete problem:

Problem 5.12. Given $\mathbf{I} \in \mathbb{C}^{N}$, find $\mathbf{H}_{h} \in \mathcal{X}_{h}$ and $\lambda_{h} \in \mathcal{L}_{h}$ such that

$$
\begin{aligned}
& a\left(\mathbf{H}_{h}, \mathbf{G}_{h}\right)+b\left(\overline{\mathbf{G}}_{h}, \lambda_{h}\right)=0 \quad \forall \mathbf{G}_{h} \in \mathcal{X}_{h}, \\
& b\left(\mathbf{H}_{h}, \nu_{h}\right)=\int_{\Gamma_{\mathbf{J}}} I \bar{\nu}_{h} \quad \forall \nu_{h} \in \mathcal{L}_{h} .
\end{aligned}
$$

The following theorem shows that this problem is well posed and establishes an error estimate for the approximate magnetic field under an appropriate mild assumption on the solution of Problem 4.3 .

Theorem 5.13. Given $\mathbf{I} \in \mathbb{C}^{N}$, Problem 5.12 has a unique solution $\left(\mathbf{H}_{h}, \lambda_{h}\right)$. Furthermore, if the solution $\left(\mathbf{H}_{\mathbf{I}}, \lambda_{\mathbf{I}}\right)$ of Problem 4.3 satisfies $\mathbf{H}_{\mathbf{I}} \in \mathrm{H}^{r}(\mathbf{c u r l}, \Omega)$ with $1 / 2<r \leq 1$, then the following error estimate holds true:

$$
\left\|\mathbf{H}_{\mathbf{I}}-\mathbf{H}_{h}\right\|_{\mathcal{X}} \leq C h^{r}\left\|\mathbf{H}_{\mathbf{I}}\right\|_{\mathrm{H}^{r}(\mathbf{c u r l}, \Omega)} .
$$

Proof. Since $\mathcal{L}_{h} \subset \mathcal{M}_{h}$, the inf-sup condition for $b$ of Lemma 5.4 holds true for all $\nu_{h} \in \mathcal{L}_{h}$, although not necessarily uniformly in $h$. On the other hand, $a$ is $\mathcal{X}$-elliptic in $\mathcal{X}_{h}$ with the ellipticity constant $\alpha$ of (3.2), which is independent of $h$. Then Theorem II.1.1 of [14] applies to this problem yielding existence of a unique solution of Problem 5.12 and the error estimate

$$
\left\|\mathbf{H}_{\mathbf{I}}-\mathbf{H}_{h}\right\|_{\mathcal{X}} \leq C\left(\inf _{\mathbf{G}_{h} \in \mathcal{W}_{h}(I)}\left\|\mathbf{H}_{\mathbf{I}}-\mathbf{G}_{h}\right\|_{\mathcal{X}}+\inf _{\nu_{h} \in \mathcal{L}_{h}}\left\|\lambda_{\mathbf{I}}-\nu_{h}\right\|_{\mathcal{M}}\right),
$$

with $C:=\max \left\{1+\frac{1}{\alpha}\|a\|_{\mathcal{X} \times \mathcal{X}}, \frac{1}{\alpha}\|b\|_{\mathcal{X} \times \mathcal{M}}\right\}$ and

$$
\mathcal{W}_{h}(I):=\left\{\mathbf{G}_{h} \in \mathcal{X}_{h}: \int_{\Gamma_{\mathbf{J}}} \operatorname{curl} \mathbf{G}_{h} \cdot \mathbf{n} \bar{\nu}_{h}=\int_{\Gamma_{\mathbf{J}}} I \bar{\nu}_{h} \quad \forall \nu_{h} \in \mathcal{L}_{h}\right\} .
$$

Now, if $\mathbf{H}_{\mathbf{I}} \in \mathrm{H}^{r}(\mathbf{c u r l}, \Omega)$ with $1 / 2<r \leq 1$, its Nédélec interpolant $\mathbf{H}_{\mathbf{I}}^{\mathrm{N}} \in \boldsymbol{\mathcal { X }}_{h}$ is well defined. In what follows we show that $\mathbf{H}_{\mathbf{I}}^{\mathrm{N}} \in \mathcal{W}_{h}(I)$. Then, the previous inequality, standard interpolation results, and estimates (4.3) and (5.1) allow us to conclude the theorem.

Notice that, for such $\mathbf{H}_{\mathbf{I}}$, $\operatorname{curl} \mathbf{H}_{\mathbf{I}} \cdot \mathbf{n} \in \mathrm{L}^{2}\left(\Gamma_{\mathrm{J}}\right)$. Hence, because of Lemma 5.1 $\operatorname{curl} \mathbf{H}_{\mathbf{I}}^{\mathrm{N}} \cdot \mathbf{n}=\Pi_{h}\left(\operatorname{curl} \mathbf{H}_{\mathbf{I}} \cdot \mathbf{n}\right)$ on $\Gamma_{\mathbf{J}}$. On the other hand, as shown in Remark 4.5 $\operatorname{curl} \mathbf{H}_{\mathbf{I}} \cdot \mathbf{n}=0$ on $\Gamma_{\mathbf{J}}^{0}$. Then, $\operatorname{curl} \mathbf{H}_{\mathbf{I}}^{N} \cdot \mathbf{n}=0$ on $\Gamma_{\mathbf{J}}^{0}$ too. Hence, $\forall \nu_{h} \in \mathcal{L}_{h}$ we have

$$
\begin{aligned}
\int_{\Gamma_{\mathbf{J}}} \operatorname{curl} \mathbf{H}_{\mathbf{I}}^{\mathrm{N}} \cdot \mathbf{n} \bar{\nu}_{h} & =\sum_{n=1}^{N}\left(\left.\bar{\nu}_{h}\right|_{\Gamma_{\mathbf{J}}^{n}}\right) \int_{\Gamma_{\mathbf{J}}^{n}} \operatorname{curl} \mathbf{H}_{\mathbf{I}}^{\mathrm{N}} \cdot \mathbf{n} \\
& =\sum_{n=1}^{N}\left(\left.\bar{\nu}_{h}\right|_{\Gamma_{\mathbf{J}}^{n}}\right) \int_{\Gamma_{\mathbf{J}}^{n}} \operatorname{curl} \mathbf{H}_{\mathbf{I}} \cdot \mathbf{n}=\int_{\Gamma_{\mathbf{J}}} I \bar{\nu}_{h},
\end{aligned}
$$

the latter because of Remark 4.9. Therefore $\mathbf{H}_{\mathbf{I}}^{\mathrm{N}} \in \mathcal{W}_{h}(I)$ and we conclude the proof. 
5.4. Discretizing Problem 4.7. In this case we use the finite-dimensional spaces

$$
\widetilde{\mathcal{X}}_{h}:=\mathcal{N}_{h}(\Omega) \quad \text { and } \quad \widetilde{\mathcal{L}}_{h}:=\left\{\nu_{h} \in \mathcal{Q}_{h}^{0}\left(\Gamma_{\mathbf{J}}\right):\left.\nu_{h}\right|_{\Gamma_{\mathbf{J}}^{n}}=\text { constant, } n=1, \ldots, N\right\}
$$

to define the following discrete problem:

Problem 5.14. Given $\mathbf{I} \in \mathbb{C}^{N}$, find $\mathbf{H}_{h} \in \widetilde{\mathcal{X}}_{h}$ and $\lambda_{h} \in \widetilde{\mathcal{L}}_{h}$ such that

$$
\begin{aligned}
& a\left(\mathbf{H}_{h}, \mathbf{G}_{h}\right)+b\left(\overline{\mathbf{G}}_{h}, \lambda_{h}\right)=0 \quad \forall \mathbf{G}_{h} \in \widetilde{\mathcal{X}}_{h}, \\
& b\left(\mathbf{H}_{h}, \nu_{h}\right)=\int_{\Gamma_{\mathbf{J}}} I \bar{\nu}_{h} \quad \forall \nu_{h} \in \widetilde{\mathcal{L}}_{h} .
\end{aligned}
$$

Since $\widetilde{\mathcal{L}}_{h} \subset \widetilde{\mathcal{M}}_{h}$, the inf-sup condition for $b$ of Lemma 5.9 holds true for all $\nu_{h} \in \widetilde{\mathcal{L}}_{h}$ uniformly in $h$; that is,

$$
\sup _{\mathbf{G}_{h} \in \widetilde{\mathcal{X}}_{h}} \frac{\left|b\left(\mathbf{G}_{h}, \nu_{h}\right)\right|}{\left\|\mathbf{G}_{h}\right\|_{\widetilde{\mathcal{X}}}} \geq \widetilde{\beta}_{*}\left\|\nu_{h}\right\|_{\widetilde{\mathcal{M}}} \quad \forall \nu_{h} \in \widetilde{\mathcal{L}}_{h}
$$

On the other hand, $a$ is $\mathcal{X}$-elliptic in $\widetilde{\mathcal{X}}_{h}$. Then, since $\widetilde{\mathcal{X}}_{h}$ is finite-dimensional, $a$ is $\widetilde{\mathcal{X}}$-elliptic in $\widetilde{\mathcal{X}}_{h}$ too, although with an ellipticity constant which may depend on $h$; namely, $\exists \alpha_{h}>0$ such that

$$
\left|a\left(\mathbf{G}_{h}, \mathbf{G}_{h}\right)\right| \geq \alpha_{h}\left\|\mathbf{G}_{h}\right\|_{\widetilde{\mathcal{X}}}^{2} \quad \forall \mathbf{G}_{h} \in \widetilde{\mathcal{X}}_{h} .
$$

Thus, Babuška-Brezzi theory can be applied to prove that the discrete problem above is well posed:

Theorem 5.15. Given $\mathbf{I} \in \mathbb{C}^{N}$, Problem 5.14 has a unique solution $\left(\mathbf{H}_{h}, \lambda_{h}\right)$.

We cannot use the standard results to obtain error estimates for the solution of this problem, because the ellipticity of $a$ is not necessarily uniform in $h$. However, we can modify conveniently the proof of Theorem II.1.1 of [14] to adapt it to this case.

Let

$$
\widetilde{\mathcal{W}}_{h}(I):=\left\{\mathbf{G}_{h} \in \widetilde{\mathcal{X}}_{h}: \int_{\Gamma_{\mathbf{J}}} \operatorname{curl} \mathbf{G}_{h} \cdot \mathbf{n} \bar{\nu}_{h}=\int_{\Gamma_{\mathbf{J}}} I \bar{\nu}_{h} \quad \forall \nu_{h} \in \widetilde{\mathcal{L}}_{h}\right\}
$$

and

$$
\widetilde{\mathcal{W}}_{h}(0):=\left\{\mathbf{G}_{h} \in \widetilde{\mathcal{X}}_{h}: \int_{\Gamma_{\mathbf{J}}} \operatorname{curl} \mathbf{G}_{h} \cdot \mathbf{n} \bar{\nu}_{h}=0 \quad \forall \nu_{h} \in \widetilde{\mathcal{L}}_{h}\right\} .
$$

We have the following result (we recall that $\widetilde{\mathcal{W}}(0)$ was defined in Section 4.2):

Lemma 5.16. $\widetilde{\mathcal{W}}_{h}(0) \subset \widetilde{\mathcal{W}}(0)$ holds.

Proof. Let $\mathbf{G}_{h} \in \widetilde{\mathcal{W}}_{h}(0)$. Since $\left.\operatorname{curl} \mathbf{G}_{h} \cdot \mathbf{n}\right|_{\Gamma_{\mathbf{J}}} \in \mathcal{Q}_{h}^{0}\left(\Gamma_{\mathbf{J}}\right)$, we have that $\operatorname{curl} \mathbf{G}_{h} \cdot \mathbf{n}=$ 0 on $\Gamma_{\mathbf{J}}^{0}$ and $\int_{\Gamma_{\mathbf{J}}^{n}} \operatorname{curl}_{\mathbf{G}} \cdot \mathbf{n}=0, n=1, \ldots, N$. Hence, $\forall \nu \in \widetilde{\mathcal{L}}$, we have

$$
\int_{\Gamma_{\mathbf{J}}} \operatorname{curl} \mathbf{G}_{h} \cdot \mathbf{n} \bar{\nu}=\sum_{n=1}^{N}\left(\left.\bar{\nu}\right|_{\Gamma_{\mathbf{J}}^{n}}\right) \int_{\Gamma_{\mathbf{J}}^{n}} \operatorname{curl} \mathbf{G}_{h} \cdot \mathbf{n}=0 .
$$

Therefore, since $\mathbf{G}_{h} \in \widetilde{\mathcal{X}}_{h} \subset \widetilde{\mathcal{X}}, \mathbf{G}_{h} \in \widetilde{\mathcal{W}}(0)$.

Now we are in order to establish an error estimate: 
Theorem 5.17. Given $\mathbf{I} \in \mathbb{C}^{N}$, let us assume that the solution $\left(\mathbf{H}_{\mathbf{I}}, \lambda_{\mathbf{I}}\right)$ of Problem 4.3 satisfies $\mathbf{H}_{\mathbf{I}} \in \mathrm{H}^{r}(\mathbf{c u r l}, \Omega)$ with $1 / 2<r \leq 1$. Then, $\left(\mathbf{H}_{\mathbf{I}}, \lambda_{\mathbf{I}}\right)$ is the solution of Problem 4.7 too and, if $\left(\mathbf{H}_{h}, \lambda_{h}\right)$ is the solution of Problem 5.14 the following error estimate holds:

$$
\left\|\mathbf{H}_{\mathbf{I}}-\mathbf{H}_{h}\right\|_{\mathcal{X}}+\left\|\lambda_{\mathbf{I}}-\lambda_{h}\right\|_{\widetilde{\mathcal{M}}} \leq C h^{r}\left\|\mathbf{H}_{\mathbf{I}}\right\|_{\mathrm{H}^{r}(\mathbf{c u r l}, \Omega)} .
$$

Proof. For $\mathbf{H}_{\mathbf{I}} \in \mathrm{H}^{r}(\operatorname{curl}, \Omega)$ with $1 / 2<r \leq 1$, $\operatorname{curl} \mathbf{H}_{\mathbf{I}} \cdot \mathbf{n} \in \mathrm{L}^{2}\left(\Gamma_{\mathbf{J}}\right)$ and, hence, $\left(\mathbf{H}_{\mathbf{I}}, \lambda_{\mathbf{I}}\right)$ is the solution of Problem 4.7 because of Theorem 4.8.

Let $\mathbf{H}_{\mathbf{I}}^{\mathrm{N}} \in \widetilde{\mathcal{X}}_{h}$ be the Nédélec interpolant of $\mathbf{H}_{\mathbf{I}}$. An argument similar to that used in the proof of Theorem 5.13 allows us to prove that $\mathbf{H}_{\mathbf{I}}^{\mathrm{N}} \in \widetilde{\mathcal{W}}_{h}(I)$. Hence, $\mathbf{H}_{h}-\mathbf{H}_{\mathbf{I}}^{\mathrm{N}} \in \widetilde{\mathcal{W}}_{h}(0)$ and, because of Lemma 5.16 $\mathbf{H}_{h}-\mathbf{H}_{\mathbf{I}}^{\mathrm{N}} \in \widetilde{\mathcal{W}}(0)$ too. Therefore, by taking $\mathbf{H}_{h}-\mathbf{H}_{\mathbf{I}}^{\mathrm{N}}$ as test function in Problems 4.7 and 5.14 we obtain

$$
a\left(\mathbf{H}_{h}-\mathbf{H}_{\mathbf{I}}^{\mathrm{N}}, \mathbf{H}_{h}-\mathbf{H}_{\mathbf{I}}^{\mathrm{N}}\right)=a\left(\mathbf{H}_{\mathbf{I}}-\mathbf{H}_{\mathbf{I}}^{\mathrm{N}}, \mathbf{H}_{h}-\mathbf{H}_{\mathbf{I}}^{\mathrm{N}}\right) .
$$

Then, from the continuity and ellipticity of $a$ in $\mathcal{X}$ (see (3.2)), we have

$$
\left\|\mathbf{H}_{h}-\mathbf{H}_{\mathbf{I}}^{\mathrm{N}}\right\|_{\mathcal{X}} \leq \frac{1}{\alpha}\|a\|_{\mathcal{X} \times \mathcal{X}}\left\|\mathbf{H}_{\mathbf{I}}-\mathbf{H}_{\mathbf{I}}^{\mathrm{N}}\right\|_{\mathcal{X}}
$$

which combined with the triangle inequality and (5.1) yield

$$
\left\|\mathbf{H}_{\mathbf{I}}-\mathbf{H}_{h}\right\|_{\mathcal{X}} \leq\left(1+\frac{1}{\alpha}\|a\|_{\mathcal{X} \times \mathcal{X}}\right)\left\|\mathbf{H}_{\mathbf{I}}-\mathbf{H}_{\mathbf{I}}^{\mathrm{N}}\right\|_{\mathcal{X}} \leq C h^{r}\left\|\mathbf{H}_{\mathbf{I}}\right\|_{\mathrm{H}^{r}(\mathbf{c u r l}, \Omega)} .
$$

On the other hand, by taking the same test function $\mathbf{G}_{h} \in \widetilde{\mathcal{X}}_{h} \subset \widetilde{\mathcal{X}}$ in Problems 4.7 and 5.14 we obtain

$$
b\left(\overline{\mathbf{G}}_{h}, \lambda_{h}-\nu_{h}\right)=a\left(\mathbf{H}_{\mathbf{I}}-\mathbf{H}_{h}, \mathbf{G}_{h}\right)+b\left(\overline{\mathbf{G}}_{h}, \lambda_{\mathbf{I}}-\nu_{h}\right) \quad \forall \nu_{h} \in \widetilde{\mathcal{L}}_{h} \quad \forall \mathbf{G}_{h} \in \widetilde{\mathcal{X}}_{h} .
$$

Then, in particular for $\nu_{h}=\Pi_{h} \lambda_{\mathbf{I}} \in \widetilde{\mathcal{L}}_{h}$ we have from (5.4)

$$
\begin{aligned}
\left\|\lambda_{h}-\Pi_{h} \lambda_{\mathbf{I}}\right\|_{\widetilde{\mathcal{M}}} & \leq \frac{1}{\widetilde{\beta}_{*}} \sup _{\mathbf{G}_{h} \in \widetilde{\mathcal{X}}_{h}} \frac{\left|b\left(\mathbf{G}_{h}, \lambda_{h}-\Pi_{h} \lambda_{\mathbf{I}}\right)\right|}{\left\|\mathbf{G}_{h}\right\|_{\widetilde{\mathcal{X}}}} \\
& \leq \frac{1}{\widetilde{\beta}_{*}}\left(\|a\|_{\mathcal{X} \times \mathcal{X}}\left\|\mathbf{H}_{\mathbf{I}}-\mathbf{H}_{h}\right\|_{\mathcal{X}}+\|b\|_{\widetilde{\mathcal{X}} \times \widetilde{\mathcal{M}}}\left\|\lambda_{\mathbf{I}}-\Pi_{h} \lambda_{\mathbf{I}}\right\|_{\widetilde{\mathcal{M}}}\right),
\end{aligned}
$$

where we have used that $a$ is continuous on $\mathcal{X} \times \mathcal{X}$ and $b$ in $\widetilde{\mathcal{X}} \times \widetilde{\mathcal{M}}$ and that $\left\|\mathbf{G}_{h}\right\|_{\mathcal{X}} \leq\left\|\mathbf{G}_{h}\right\|_{\widetilde{\mathcal{X}}}$. Hence, from the triangle inequality we obtain

$$
\left\|\lambda_{\mathbf{I}}-\lambda_{h}\right\|_{\widetilde{\mathcal{M}}} \leq\left(1+\frac{1}{\widetilde{\beta}_{*}}\|b\|_{\widetilde{\mathcal{X}} \times \widetilde{\mathcal{M}}}\right)\left\|\lambda_{\mathbf{I}}-\Pi_{h} \lambda_{\mathbf{I}}\right\|_{\widetilde{\mathcal{M}}}+\frac{1}{\widetilde{\beta}_{*}}\|a\|_{\mathcal{X} \times \boldsymbol{X}}\left\|\mathbf{H}_{\mathbf{I}}-\mathbf{H}_{h}\right\|_{\mathcal{X}},
$$

and we conclude the proof from this inequality, standard error estimates for the $\mathrm{L}^{2}\left(\Gamma_{\mathrm{J}}\right)$-projection, and estimates (4.3) and (5.5).

\section{Numerical Results}

In this section we present some numerical results obtained with MaTLAB codes developed by us, which implement the different methods described above. We have solved a particular test problem with known analytical solution to validate the computer codes and to assess the performance and convergence properties of each method.

We have solved the eddy current problem in a cylindrical domain $\Omega$ of radius $R$ and height $L$, which is a bounded section of an infinite cylinder (see Figure 2). 


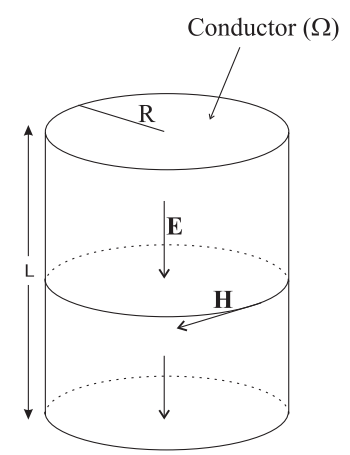

$I(t)=I_{1} \cos (\omega t)$

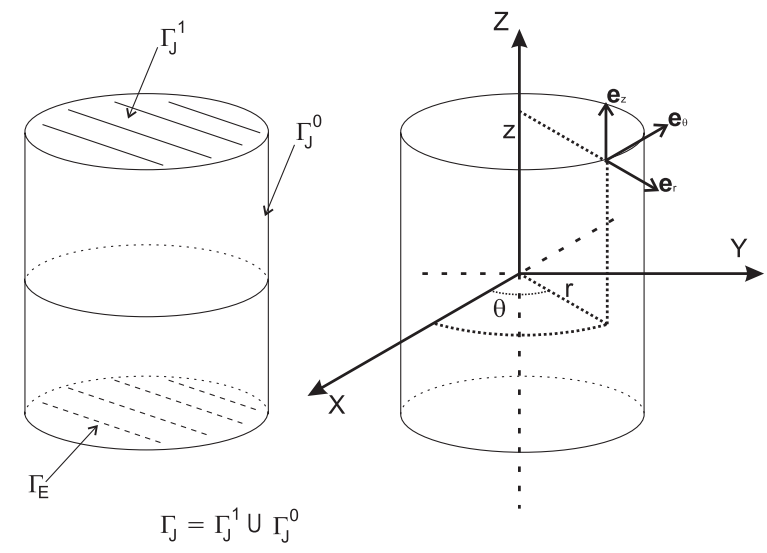

Figure 2. Sketch of the domain. Coordinate system.

We have considered an alternating current $\mathbf{J}$ going through the conductor $\Omega$ in the direction of its axis. This current has been assumed to be axially symmetric with an intensity $I(t)=I_{1} \cos (\omega t)$. Thus, we have taken the bottom surface of the cylinder as the current exit boundary $\Gamma_{\mathbf{E}}$, its lateral surface as the current density flux-free boundary $\Gamma_{\mathrm{J}}^{0}$, and its top as the input current boundary $\Gamma_{\mathrm{J}}^{1}$. Concerning the physical properties, the electric conductivity $\sigma$ and the magnetic permeability $\mu$ are taken as constants in $\Omega$.

We have used the following geometrical and physical data:

- $R=0.5 \mathrm{~m}$;

- $L=1 \mathrm{~m}$;

- $\sigma=10^{6}(\Omega \mathrm{m})^{-1}$

- $\mu=\mu_{0}=4 \pi 10^{-7} \mathrm{Hm}^{-1}$ (magnetic permeability of free space);

- $I_{1}=7 \times 10^{4} \mathrm{~A}$

- $\omega=2 \pi \times 50 \mathrm{~Hz}$.

To obtain the analytical solution of this problem, we consider a cylindrical coordinate system $(r, \theta, z)$ with the $z$-axis coinciding with the axis of the cylinder (see Figure 2). We denote $\mathbf{e}_{r}, \mathbf{e}_{\theta}$, and $\mathbf{e}_{z}$ the unit vectors in the corresponding coordinate directions.

Because of the conditions assumed on $\mathbf{J}$, only the $z$-component of the electric field $\mathbf{E}=\frac{1}{\sigma} \mathbf{J}$ does not vanish in the conductor. Moreover, it depends on the radial coordinate $r$ but is independent of the other two coordinates $\theta$ and $z$. Consequently, only the $\theta$-component of the magnetic field $\mathbf{H}=\frac{\mathrm{i}}{\omega \mu} \mathbf{c u r l} \mathbf{E}$ does not vanish and it also depends only on the coordinate $r$. Then, after writing the curl operator in cylindrical coordinates, straightforward computations allow us to obtain the following expression for the magnetic and electric field (see [7] for details):

$$
\left.\begin{array}{l}
\mathbf{H}(r, \theta, z)=-\frac{I_{1}}{2 \pi R} \frac{\mathcal{I}_{1}(\gamma r)}{\mathcal{I}_{1}(\gamma R)} \mathbf{e}_{\theta}, \\
\mathbf{E}(r, \theta, z)=-\frac{I_{1} \gamma}{2 \pi R \sigma} \frac{\mathcal{I}_{0}(\gamma r)}{\mathcal{I}_{1}(\gamma R)} \mathbf{e}_{z},
\end{array}\right\} \quad r \in[0, R], \theta \in[0,2 \pi], 0 \leq z \leq L,
$$

where $\mathcal{I}_{1}$ and $\mathcal{I}_{0}$ are the modified Bessel functions of the first kind and orders 1 and 0 , respectively, and $\gamma=\sqrt{i \omega \mu \sigma} \in \mathbb{C}$. 


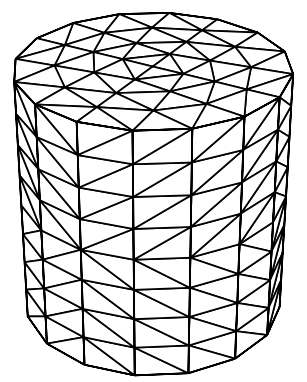

Figure 3. Coarsest mesh.

On the other hand, since $\mathbf{E} \times \mathbf{n}=-\nabla \bar{\lambda} \times \mathbf{n}$ on $\Gamma_{\mathrm{J}}$, after writing the gradient operator in cylindrical coordinates, we obtain the following analytical expression for the Lagrange multiplier $\lambda$ on $\Gamma_{\mathbf{J}}$ :

$$
\lambda(r, \theta, z)=z C, \quad \text { with } \quad C=\frac{I_{1} \bar{\gamma}}{2 \pi R \sigma} \frac{\mathcal{I}_{0}(\bar{\gamma} R)}{\mathcal{I}_{1}(\bar{\gamma} R)} .
$$

Notice that $\lambda$ is constant on the top and bottom surfaces $\Gamma_{J}^{1}$ and $\Gamma_{E}$, respectively.

Thus, this problem can be used as a test for both sets of boundary conditions (2.9) -(2.11) and (2.4)-(2.8). In the first case, a direct computation leads to the following expression for the boundary data $g=\left.\operatorname{curl} \mathbf{H} \cdot \mathbf{n}\right|_{\Gamma_{\mathbf{J}}}$ in (2.10),

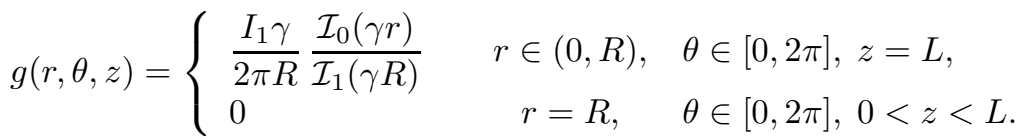

In the second case, the input current intensity $I_{1}$ in (2.5) is just the intensity amplitude of the alternating current $I(t)=I_{1} \cos (\omega t)$ imposed through $\Omega$.

Notice that the four proposed finite element methods are applicable to this problem, since $\operatorname{curl} \mathbf{H} \cdot \mathbf{n} \in \mathrm{L}^{2}\left(\Gamma_{\mathrm{J}}\right)$.

All of the numerical methods have been used on several successively refined meshes and we have compared the obtained numerical solutions with the analytical one. Figure 3 shows the coarsest mesh used for the domain.

Tables 1 to 4 show the norms of the approximate solutions $\mathbf{H}_{h}$ and $\lambda_{h}$ computed on several meshes and the corresponding absolute errors for all of the discrete problems analyzed in the previous section. In all tables we identify each mesh with its total number of edges which correspond to the number of degrees of freedom (d.o.f.) of $\mathbf{H}_{h}$. We include one table for each problem.

We have chosen the following energy-like norms:

$$
\begin{aligned}
& \|\mathbf{H}\|_{a, \Omega}:=|a(\mathbf{H}, \mathbf{H})|^{1 / 2} \quad \text { for the magnetic field, } \\
& |\mathbf{H}|_{\sigma, \Gamma_{\mathbf{J}}}:=\sigma^{-1 / 2}\|\mathbf{c u r l} \mathbf{H} \cdot \mathbf{n}\|_{L^{2}\left(\Gamma_{\mathbf{J}}\right)} \text { for the current density flux. }
\end{aligned}
$$

Notice that $\|\cdot\|_{a, \Omega}$ is equivalent to $\|\cdot\|_{\mathcal{X}}$, whereas $\|\cdot\|_{a, \Omega}+|\cdot|_{\sigma, \Gamma_{\mathbf{J}}}$ is equivalent to $\|\cdot\|_{\widetilde{\mathcal{X}}}$. 
TABLE 1. Solution $\left(\mathbf{H}_{h}, \lambda_{h}\right)$ of Problem 5.6.

\begin{tabular}{|c||c|c||c|c|}
\hline d.o.f. & $\left\|\mathbf{H}_{h}\right\|_{a, \Omega}$ & $\left\|\mathbf{H}-\mathbf{H}_{h}\right\|_{a, \Omega}$ & $\left\|\lambda_{h}\right\|_{\mathcal{M}}$ & $\left\|\lambda-\lambda_{h}\right\|_{\mathcal{M}}$ \\
\hline 2096 & 168.00 & 85.44 & 0.4007 & 0.0489 \\
6720 & 172.83 & 68.22 & 0.3951 & 0.0356 \\
15520 & 174.79 & 55.00 & 0.3955 & 0.0217 \\
29840 & 176.04 & 45.59 & 0.3978 & 0.0146 \\
51024 & 176.86 & 38.75 & 0.3997 & 0.0107 \\
80416 & 177.41 & 33.63 & 0.4011 & 0.0079 \\
119360 & 177.80 & 29.66 & 0.4022 & 0.0061 \\
169200 & 178.06 & 26.51 & 0.4013 & 0.0049 \\
\hline
\end{tabular}

TABle 2. Solution $\left(\mathbf{H}_{h}, \lambda_{h}\right)$ of Problem 5.8 .

\begin{tabular}{|c||c|c||c|c||c|c|}
\hline d.o.f. & $\left\|\mathbf{H}_{h}\right\|_{a, \Omega}$ & $\left\|\mathbf{H}-\mathbf{H}_{h}\right\|_{a, \Omega}$ & $\left|\mathbf{H}_{h}\right|_{\sigma, \Gamma_{\mathbf{J}}}$ & $\left|\mathbf{H}-\mathbf{H}_{h}\right|_{\sigma, \Gamma_{\mathbf{J}}}$ & $\left\|\lambda_{h}\right\|_{\widetilde{\mathcal{M}}}$ & $\left\|\lambda-\lambda_{h}\right\|_{\widetilde{\mathcal{M}}}$ \\
\hline 2096 & 167.91 & 85.54 & 117.10 & 79.87 & 0.6105 & 0.0282 \\
6720 & 172.78 & 68.26 & 134.86 & 60.25 & 0.6031 & 0.0347 \\
15520 & 174.78 & 55.03 & 142.39 & 47.32 & 0.6045 & 0.0271 \\
29840 & 176.04 & 45.61 & 146.18 & 38.69 & 0.6079 & 0.0203 \\
51024 & 176.86 & 38.77 & 148.33 & 32.63 & 0.6108 & 0.0155 \\
80416 & 177.41 & 33.63 & 149.66 & 28.18 & 0.6130 & 0.0123 \\
119360 & 177.79 & 29.66 & 150.58 & 24.77 & 0.6146 & 0.0100 \\
169200 & 178.06 & 26.51 & 151.14 & 22.09 & 0.6157 & 0.0084 \\
\hline
\end{tabular}

TABLE 3. Solution $\left(\mathbf{H}_{h}, \lambda_{h}\right)$ of Problem 5.12

\begin{tabular}{|c||c|c||c|c|}
\hline d.o.f. & $\left\|\mathbf{H}_{h}\right\|_{a, \Omega}$ & $\left\|\mathbf{H}-\mathbf{H}_{h}\right\|_{a, \Omega}$ & $\left\|\lambda_{h}\right\|_{\mathcal{M}}$ & $\left\|\lambda-\lambda_{h}\right\|_{\mathcal{M}}$ \\
\hline 2096 & 189.80 & 105.79 & 0.4522 & 0.1538 \\
6720 & 181.31 & 74.62 & 0.4143 & 0.0794 \\
15520 & 179.30 & 57.70 & 0.4059 & 0.0470 \\
29840 & 178.65 & 46.96 & 0.4042 & 0.0307 \\
51024 & 178.78 & 39.54 & 0.4041 & 0.0215 \\
80416 & 178.81 & 34.12 & 0.4044 & 0.0159 \\
119360 & 178.85 & 29.99 & 0.4047 & 0.0122 \\
169200 & 178.90 & 26.74 & 0.4049 & 0.0097 \\
\hline
\end{tabular}

TABLE 4. Solution $\left(\mathbf{H}_{h}, \lambda_{h}\right)$ of Problem 5.14

\begin{tabular}{|c||c|c||c|c||c|c|}
\hline d.o.f. & $\left\|\mathbf{H}_{h}\right\|_{a, \Omega}$ & $\left\|\mathbf{H}-\mathbf{H}_{h}\right\|_{a, \Omega}$ & $\left|\mathbf{H}_{h}\right|_{\sigma, \Gamma_{\mathbf{J}}}$ & $\left|\mathbf{H}-\mathbf{H}_{h}\right|_{\sigma, \Gamma_{\mathbf{J}}}$ & $\left\|\lambda_{h}\right\|_{\widetilde{\mathcal{M}}}$ & $\left\|\lambda-\lambda_{h}\right\|_{\widetilde{\mathcal{M}}}$ \\
\hline 2096 & 189.78 & 105.93 & 132.77 & 79.54 & 0.6904 & 0.2286 \\
6720 & 181.30 & 74.66 & 140.98 & 59.99 & 0.6332 & 0.1188 \\
15520 & 179.30 & 57.72 & 145.54 & 47.22 & 0.6204 & 0.0708 \\
29840 & 178.85 & 46.98 & 148.10 & 38.69 & 0.6178 & 0.0466 \\
51024 & 178.78 & 39.55 & 149.62 & 32.68 & 0.6175 & 0.0320 \\
80416 & 178.81 & 34.13 & 150.58 & 28.24 & 0.6179 & 0.0246 \\
119360 & 178.85 & 29.99 & 151.23 & 24.84 & 0.6183 & 0.0191 \\
169200 & 178.90 & 26.74 & 151.68 & 22.17 & 0.6187 & 0.0153 \\
\hline
\end{tabular}




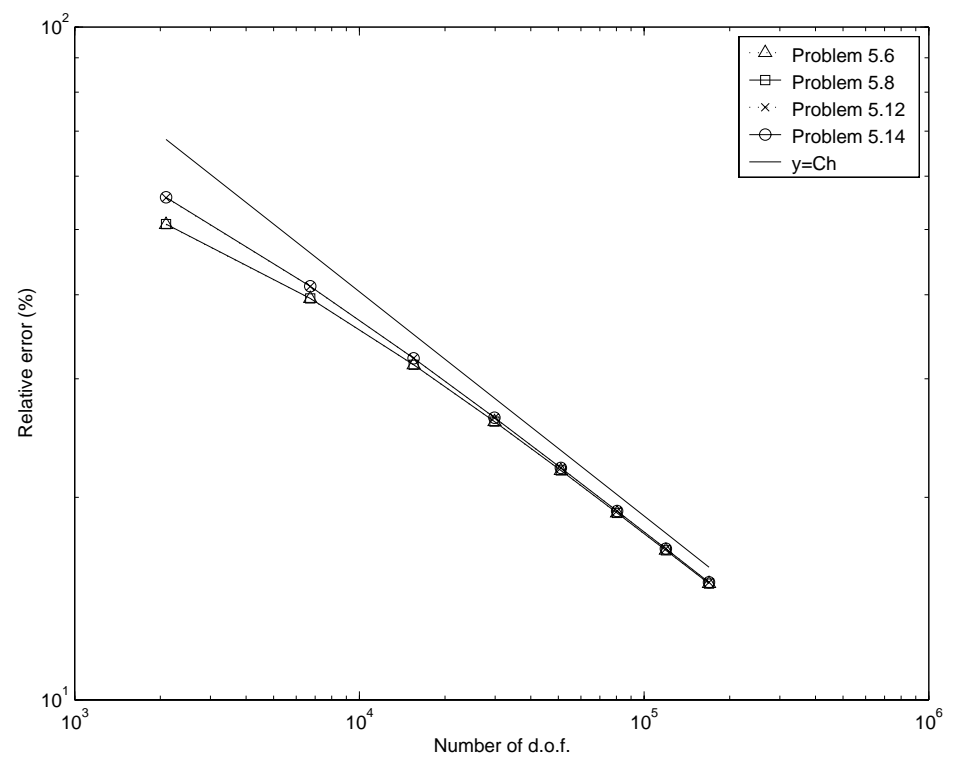

Figure 4. Error curve for $\mathbf{H}_{h}$ (log-log scale). Case of physical constants.

On the other hand, to compute the $\mathcal{M}$ norm (i.e., $\mathrm{H}_{00}^{1 / 2}\left(\Gamma_{\mathrm{J}}\right)$ norm) involved in Problems 5.6 and 5.12 we have used the following characterization: for $\nu \in$ $\mathrm{H}_{00}^{1 / 2}\left(\Gamma_{\mathbf{J}}\right)$, there exists a unique $u_{\nu} \in \mathrm{H}_{\Gamma_{\mathbf{E}}}^{1}(\Omega)$ satisfying

$$
\begin{aligned}
-\Delta u_{\nu}=0 & \text { in } \Omega, \\
u_{\nu}=\nu & \text { on } \Gamma_{J},
\end{aligned}
$$

and $\left\|\nabla u_{\nu}\right\|_{\mathrm{L}^{2}(\Omega)^{3}}$ is equivalent to the norm $\|\nu\|_{\mathrm{H}_{00}^{1 / 2}\left(\Gamma_{\mathrm{J}}\right)}$. In our test, it is simple to verify that $u_{\lambda}=\lambda$ in the whole $\Omega$, with $\lambda$ given by (6.1). Then, to compute the $\mathcal{M}$ norm of a numerical solution $\lambda_{h}$ and its corresponding error, we have solved by a standard finite element method the problem above with $\nu=\lambda_{h}$.

Notice that the computed magnetic fields $\mathbf{H}_{h}$ and Lagrange multipliers $\lambda_{h}$ converge for all the methods.

Figures 4 and 5 show log-log plots of the errors for the computed magnetic fields $\mathbf{H}_{h}$ and Lagrange multipliers $\lambda_{h}$, respectively, versus the number of degrees of freedom, for the same meshes and the four methods. We have used the norm $\|\cdot\|_{a \Omega \Omega}$ for the former and the $\mathrm{L}^{2}\left(\Gamma_{\mathrm{J}}\right)$ norm for the latter.

A linear dependence on the mesh size can be clearly observed for all $\mathbf{H}_{h}$. However, for the Lagrange multiplier $\lambda_{h}$ the conclusions are not clear. Figure 5 shows an almost quadratic dependence on the mesh size which could not be true, at least for Problems 5.8 and 5.14 where $\lambda$ is approximated by piecewise constant functions. Since in three-dimensional experiments the mesh size cannot become very small because of computer limitations, we cannot confirm the actual order of convergence of the Lagrange multiplier with this example. This is mainly due to the large magnitude of some of the physical constants.

To avoid this drawback, we have solved a similar problem with all of the physical constants set equal to one, namely, $\sigma=\mu=\omega=I_{1}=1$. Figure 6 shows a log-log 


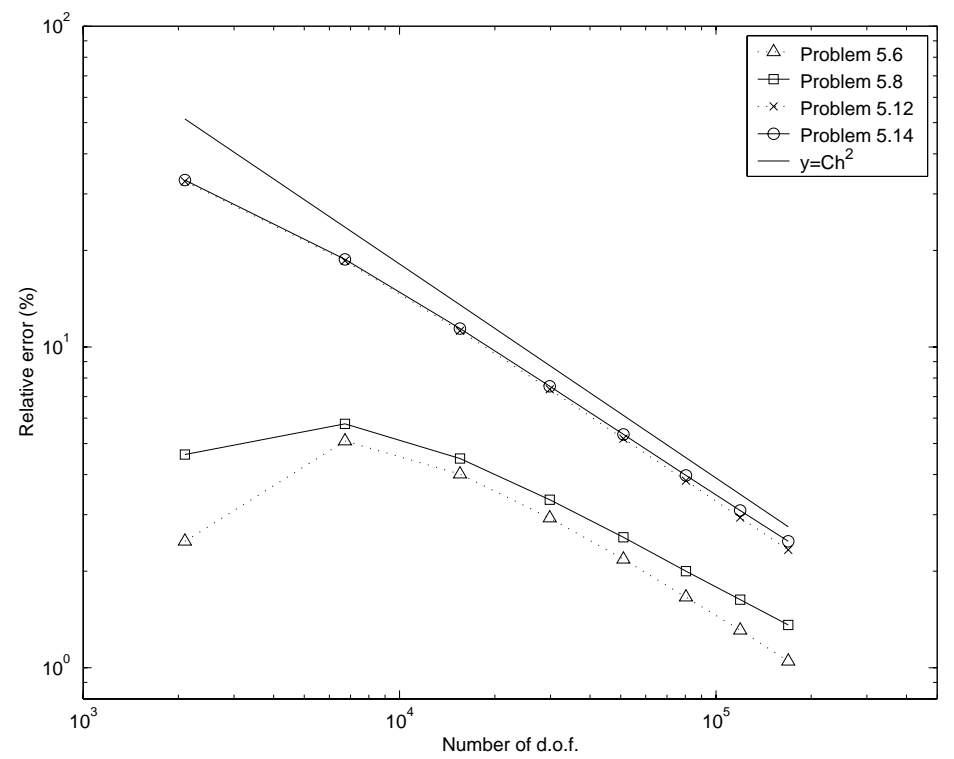

FiguRE 5. Error curve for $\lambda_{h}$ (log-log scale). Case of physical constants.

plot of the relative errors for the Lagrange multipliers computed with each of the methods. Now, a quadratic dependence on the mesh size can be observed for the methods approximating the Lagrange multiplier with piecewise linear functions, while a linear dependence appears for those using piecewise constant functions.

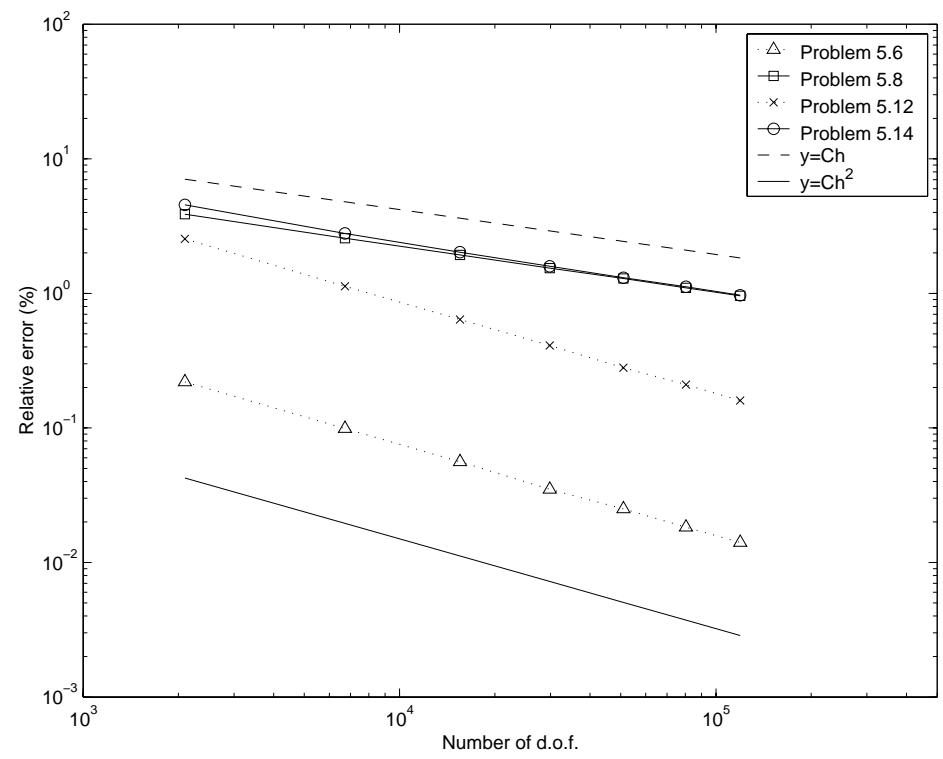

FiguRE 6. Error curve for $\lambda_{h}$ (log-log scale). Case of unit constants. 


\section{Conclusions}

We have studied the eddy current problem in a conducting bounded domain. The main point is the introduction of physically realistic boundary conditions. To handle these boundary conditions, which are neither essential nor natural, we have introduced a Lagrange multiplier defined on the boundary which leads to mixed problems. This multiplier has a physical meaning: it is a surface electric potential defined on the boundary of the domain.

Two kinds of boundary conditions have been considered:

- the current density flux is given (Problems 3.3 and 3.9);

- the current intensities are given (Problems 4.3 and 4.7).

The second boundary condition is the most interesting in practice, because it involves physical data easy to measure in real problems. The reason to consider the first boundary condition too is two-fold: on one hand, as an intermediate step for the treatment of the other case and, on the other hand, because in some problems the current density flux could be known, for instance if it is the output of other computations.

Each mixed problem involves the magnetic field and the Lagrange multiplier, and we have proposed finite element methods to obtain their numerical solution. The magnetic field has been approximated by using Nédélec edge elements in all of the problems, while the discretization of the Lagrange multiplier depends on the regularity of curl H $\cdot \mathbf{n}$. More precisely, we have used

- piecewise linear functions in the most general setting, namely, if curl $\mathbf{H} \cdot \mathbf{n} \in$ $\mathrm{H}_{00}^{-1 / 2}\left(\Gamma_{\mathrm{J}}\right)$ (Problems 5.6 and 5.12 );

- piecewise constant functions, only if $\operatorname{curl} \mathbf{H} \cdot \mathbf{n} \in \mathrm{L}^{2}\left(\Gamma_{\mathbf{J}}\right)$ (Problems 5.8 and 5.14 .

In the first case, the discrete inf-sup conditions corresponding to the mixed problems are not uniform with respect to the mesh size. Because of this, we could only prove $\mathrm{L}^{2}$ convergence for the magnetic and electric fields. Instead, in the second case, we have proved $\mathrm{L}^{2}$ error estimates for the multiplier too.

Both discretizations of the Lagrange multiplier have some interest in and of themselves. The first one is valid without the need of the additional smoothness assumption curl $\mathbf{H} \cdot \mathbf{n} \in \mathrm{L}^{2}\left(\Gamma_{\mathrm{J}}\right)$. The second one has the advantage that a thorough mathematical analysis including optimal order error estimates for the multipliers has been given in this paper. However, in spite of the lack of a proof for the convergence of the Lagrange multiplier, the experiments seem to indicate that an optimal order error estimate should be valid for the first discretization, too. Thus a conclusion as to which is the best choice would need further work.

\section{REFERENCES}

[1] A. Alonso P. Fernandes, and A. Valli, The time-harmonic eddy current problem in general domains: solvability via scalar potentials, Lect. Notes Comp. Sci. Engrg., 28 (2003) 143-163.

[2] A. Alonso and A. Valli, A domain decomposition approach for heterogeneous time-harmonic Maxwell equations, Comput. Methods Appl. Mech. Engrg., 143 (1997) 97-112. MR98b:78020

[3] _ An optimal domain decomposition preconditioner for low-frequency time-harmonic Maxwell equations, Math. Comp., 68 (1999) 607-631. MR99i:78002

[4] C. Amrouche, C. Bernardi, M. Dauge, and V. Girault, Vector potentials in three-dimensional non-smooth domains, Math. Meth. Appl. Sci., 21 (1998) 823-864. MR99e:35037 
[5] A. Bermúdez, J. Bullón, and F. Pena, A finite element method for the thermoelectrical modelling of electrodes, Comm. Numer. Methods Engrg., 14 (1998) 581-593.

[6] A. Bermúdez, J. Bullón, F. Pena, and P. Salgado, A numerical method for transient simulation of metallurgical compound electrodes, Finite Elem. Anal. Des., 39 (4) (2003) 283-299.

[7] A. Bermúdez, R. Rodríguez, and P. Salgado, A finite element method with Lagrange multipliers for low-frequency harmonic Maxwell equations, SIAM J. Numer. Anal., 40 (5) (2002) 1823-1849. MR 2004b:78017

[8] _ Modeling and numerical treatment of boundary data in an eddy current problem, C. R. Acad. Sci. Paris, Serie I, 335 (7) (2002) 633-638.

[9] A. Bossavit, The computation of eddy-currents in dimension 3 by using mixed finite elements and boundary elements in association, Math. Comput. Modelling, 15 (1991) 33-42. MR 92c:78001

[10] - Computational Electromagnetism. Variational Formulations, Complementarity, Edge Elements. Academic Press. San Diego, CA, 1998. MR.99m:78001

[11] _ Most general "non-local" boundary conditions for the Maxwell equation in a bounded region, COMPEL, 19 (2000) 239-245. MR2001f:78009

[12] A. Bossavit and J.C. Vérité, A mixed FEM-BIEM method to solve 3-D eddy current problems, IEEE Trans. Mag., 18 (1982) 431-435. MR2001f:78009

[13] M. Dauge, Elliptic boundary value problems on corner domains: smoothness and asymptotics of solutions, Lecture Notes in Mathematics 1341, Springer, Berlin, 1988. MR91a:35078

[14] V. Girault and P.A. Raviart, Finite Element Methods for Navier-Stokes Equations. Theory and Algorithms. Springer-Verlag, Berlin, 1986. MR88b:65129

[15] S.I. Hariharan and R.C. MacCamy, An integral equation procedure for eddy current problems, J. Comput. Phys., 45 (1982) 80-99. MR83g:78011

[16] R. Innvær and L. Olsen, Practical use of mathematical models for Soderberg electrodes. Elkem Carbon Technical Paper presented at the A.I.M.E. Conference. (1980).

[17] R.C. MacCamy and E. Stephan, A skin effect approximation for eddy current problems, Arch. Rational Mech. Anal., 90 (1985) 87-98. MR86j:78005

[18] R.C. MacCamy and M. Suri, A time-dependent interface problem for two-dimensional eddy currents, Quart. Appl. Math., 44 (1987) 675-690. MF.87m:78007

[19] J.C. Nédélec, Mixed finite elements in $\mathbb{R}^{3}$, Numer. Math., 35 (1980) 315-341. MR81k:65125

Departamento de Matemática Aplicada, Universidade de Santiago de Compostela, 15706 Santiago de Compostela, Spain

E-mail address: mabermud@usc.es

GI²MA, Departamento de Ingeniería Matemática, Universidad de Concepción, Casilla 160-C, Concepción, Chile

E-mail address: rodolfo@ing-mat.udec.cl

Departamento de Matemática Aplicada, Universidade de Santiago de Compostela, 15706 Santiago de Compostela, Spain

E-mail address: mpilar@usc.es 